\title{
CXCR4 downregulation of let-7a drives chemoresistance in acute myeloid leukemia
}

\author{
Ye Chen, ${ }^{1}$ Rodrigo Jacamo, ${ }^{1}$ Marina Konopleva, ${ }^{1,2}$ Ramiro Garzon, ${ }^{3}$ \\ Carlo Croce, ${ }^{3}$ and Michael Andreeff 1,2
}

\begin{abstract}
${ }^{1}$ Section of Molecular Hematology and Therapy, Department of Leukemia, and 2Department of Stem Cell Transplantation and Cellular Therapy, University of Texas MD Anderson Cancer Center, Houston, Texas, USA. ${ }^{3}$ The Comprehensive Cancer Center, The Ohio State University, Columbus, Ohio, USA.
\end{abstract}

\begin{abstract}
We examined the role of microRNAs (miRNAs) in targeting the stromal-derived factor $1 \alpha /$ CXCR4 (SDF-1 $\alpha /$ CXCR4) axis to overcome chemoresistance of AML cells. Microarray analysis of OCI-AML3 cells revealed that the miRNA let-7a was downregulated by SDF-1 $\alpha$-mediated CXCR4 activation and increased by CXCR4 inhibition. Overexpression of let-7a in AML cell lines was associated with decreased c-Myc and BCL-XL protein expression and enhanced chemosensitivity, both in vitro and in vivo. We identified the transcription factor Yin Yang 1 (YY1) as a link between SDF-1 $\alpha /$ CXCR4 signaling and let-7a, as YY1 was upregulated by SDF-1 $\alpha$ and downregulated by treatment with a CXCR4 antagonist. ChIP assay confirmed the binding of YY1 to unprocessed let-7a DNA fragments, and treatment with YY1 shRNA increased let-7a expression. In primary human AML samples, high CXCR4 expression was associated with low let-7a levels. Xenografts of primary human AML cells engineered to overexpress let-7a exhibited enhanced sensitivity to cytarabine, resulting in greatly extended survival of immunodeficient mice. Based on these data, we propose that CXCR4 induces chemoresistance by downregulating let-7a to promote YY1-mediated transcriptional activation of MYC and $B C L X L$ in AML cells.
\end{abstract}

\section{Introduction}

Acute myeloid leukemia (AML) is a hematologic disease characterized by dysregulated cell proliferation, impaired cell death, and accumulation of immature myeloid cells in BM and peripheral blood $(1,2)$. The disease carries an extremely poor prognosis, and the principal cause of treatment failure is chemotherapy resistance $(2,3)$. Leukemic cells have been shown to hijack the homeostatic mechanisms of normal hematopoietic stem cells and take refuge within the BM niche $(4,5)$. This mechanism is pivotal to the survival of residual cells after chemotherapy and consequently contributes to disease relapse.

In recent years, interrupting the connection between leukemic cells and the tumor microenvironment by targeting the stromal-derived factor $1 \alpha /$ CXCR 4 (SDF- $1 \alpha /$ CXCR4) axis has become an attractive therapeutic approach for AML. Our group and others have shown that culturing of AML cells with SDF$1 \alpha$ (also known as CXCL12) promotes their survival, whereas adding neutralizing CXCR4 antibodies, SDF-1 $\alpha$ antibodies, or the CXCR4 inhibitor AMD3100 significantly decreases it. BMderived mesenchymal stromal cells can also protect AML cells from chemotherapeutic drug-induced apoptosis $(6,7)$. Moreover, weekly administration of anti-human CXCR4 antibody to mice previously engrafted with human AML cells leads to a dramatic decrease of human AML cells in BM, blood, and spleen in a dose- and time-dependent manner $(8,9)$. However, the mechanisms involved in this SDF- $1 \alpha / C X C R 4$ axis-targeting progress are not fully understood.

microRNAs (miRNAs) are small noncoding regulatory RNAs approximately $18-25$ nucleotides in length $(10,11)$. Each miRNA has the potential to target a large number of genes. The discovery of miRNAs in the early 1990s opened a new era in under-

Conflict of interest: The authors have declared that no conflict of interest exists. Citation for this article: J Clin Invest. 2013;123(6):2395-2407. doi:10.1172/JCI66553. standing transcriptional and posttranscriptional regulation of gene expression in biological processes (11-13). miRNAs are now known to play roles in almost all aspects of cancer biology, including proliferation, apoptosis, invasion and metastasis, and angiogenesis (14-16). In recent years, functional and prognostic studies have confirmed that miRNAs plays an active role in hematologic malignancies, and some miRNAs have been proposed as prognostic markers and therapeutic targets in leukemia treatment. Calin et al. found that $65 \%$ of B cell chronic lymphocytic leukemia patients have deletions of chromosome 13q14, a locus that includes miR-15a and miR-16-1, which consequently present downregulated expression (17). Garzon et al. reported that miRNAs including miR-15a, miR-15b, miR-16-1, miR-223, miR-342, and miR-107 are upregulated, whereas miR-181b is downregulated, in acute promyelocytic leukemia (18). miR-155 was reported to be upregulated in patients with an internal tandem duplication of the FLT3 gene (19). Schotte et al. showed that 14 miRNAs are upregulated (miR-128a, miR-142-3p, miR-142-5p, miR-150, miR181a, miR-181b, miR-181c, miR-193a, miR-196b, miR-30e-5p, miR-34b, miR-365, miR-582, and miR-708), and 5 downregulated (miR-100, miR-125b, miR-151-5p, miR-99a, and let-7e), in acute lymphoblastic leukemia cells compared with normal CD34+ cells (20). Upregulation of miR-181a and miR-335 has been observed in AML patients carrying CEBPA gene mutations (21, 22). And, very recently, miR-3151 was reported to independently affect the outcome of patients with cytogenetically normal AML (23).

In the present study, we analyzed the mechanisms of CXCR4 signaling-mediated chemoresistance and demonstrated that the human miRNA let-7a, which negatively regulates BCL-XL expression, is regulated by SDF- $1 \alpha / C X C R 4$ signaling in human AML cells. In our experiments, inhibiting CXCR4 or overexpressing let-7a in AML cells led to reduced expression of BCL-XL and enhanced cytarabine-induced (Ara-C-induced) apoptosis both in vitro and in vivo. 
A

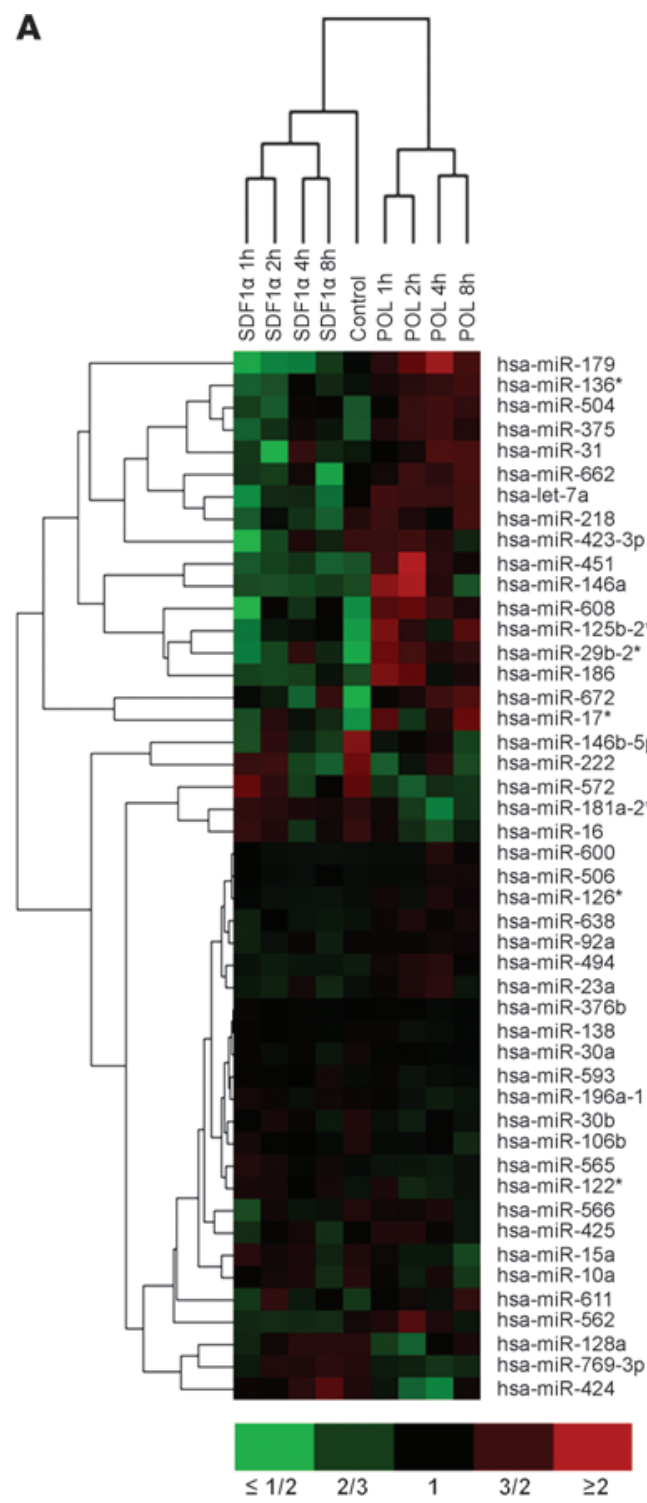

B

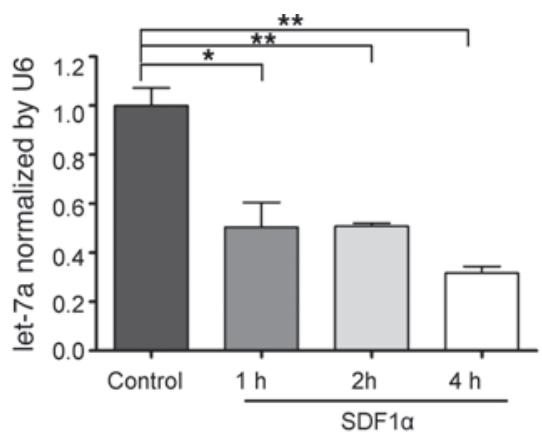

C

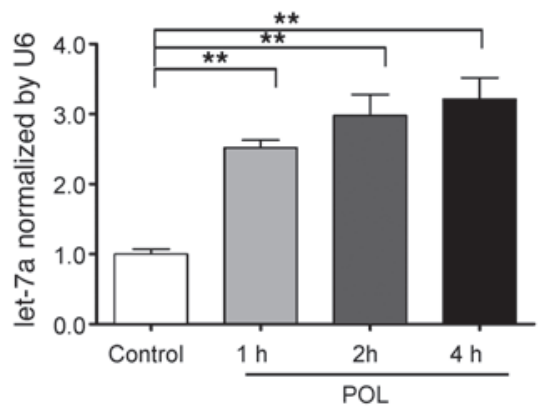

D

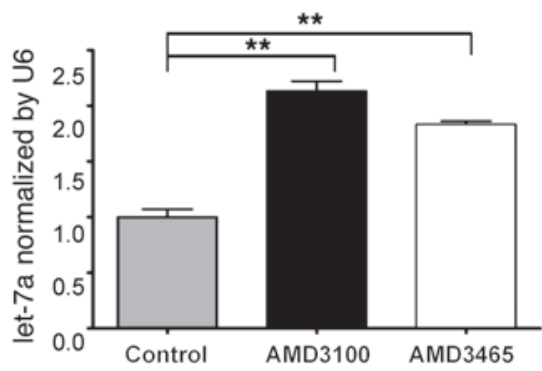

Figure 1

CXCR4-mediated signaling influences miRNA profiles in OCI-AML3 cells. (A) OCI-AML3 cells were treated with SDF-1 $\alpha$ or POL6326, total RNA was extracted at specific time points, and mRNA expression profiling was performed. 47 miRNA probes were identified to be significantly changed in either direction with treatment. (B and C) qRT-PCR was performed to evaluate let-7a expression in response to SDF-1 $\alpha$ (B) or POL6326 (C) treatment for the indicated times. (D) Upregulation of let-7a was also obtained in OCI-AML3 cells with administration of AMD CXCR4 inhibitors. ${ }^{\star} P<0.05,{ }^{* *} P<0.01$.

\section{Results}

let-7a in OCI-AML3 cells is downregulated by SDF-1 $\alpha$ treatment and upregulated with CXCR4 antagonist. To explore how CXCR4-mediated signaling in AML cells elicits chemoresistance, we first performed a miRNA microarray platform (see Methods). OCI-AML3 cells were treated with $100 \mathrm{ng} / \mathrm{ml} \mathrm{SDF}-1 \alpha$ or $250 \mathrm{nM}$ POL6326 (a CXCR4 antagonist), total RNA was extracted at specific time points $(0,1,2,4$, and 8 hours), and miRNA expression profiling was performed. 47 miRNA probes were identified to be significantly changed in either direction with treatment (significant at 0.01 level of the univariate test; Figure 1A). We focused on miRNAs that could potentially function as tumor suppressors and connect CXCR4 signaling to chemoresistance. The let-7a coding sequence was selected for further analysis because it was not only one of the most strongly downregulated miRNAs in OCI-AML3 cells after SDF-1 $\alpha$ treatment, but was also highly elevated when OCI-AML3 cells were treated with the CXCR4 antagonist. The results were confirmed by real-time quantitative RT-PCR (qRT-PCR; Figure 1, $\mathrm{B}$ and $\mathrm{C}$ ). In addition, the structurally unrelated CXCR4 inhibitors AMD3100 and AMD3465 showed similar effects on let-7a levels in OCI-AML3 cells (Figure 1D). Together, these observations indicated that CXCR4-mediated signaling influences miRNA profiles in OCI-AML3 cells.

Transfection of synthetic let- 7 a sensitizes OCI-AML3 cells to Ara-C. To investigate the effect of let-7a in leukemic cells on chemotherapyinduced apoptosis, we transfected let-7a mimic $(0.25 \mu \mathrm{M})$ or nega- 
A

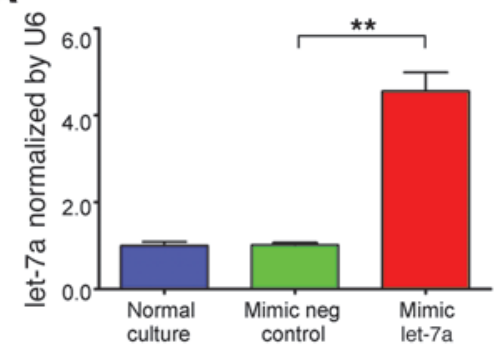

B

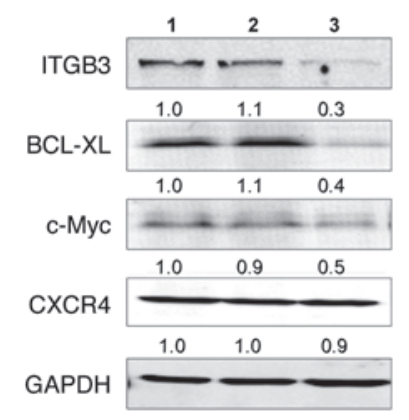

C

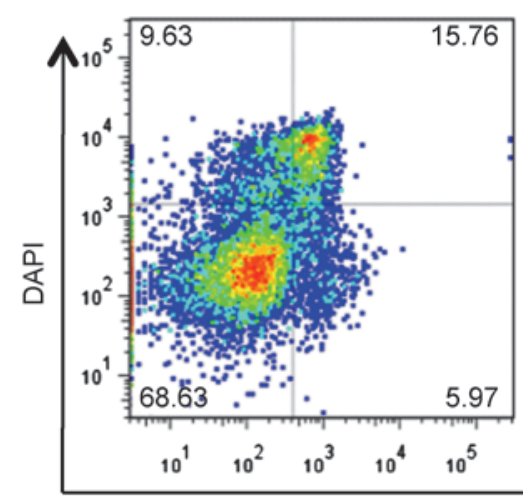

Mimic negative control

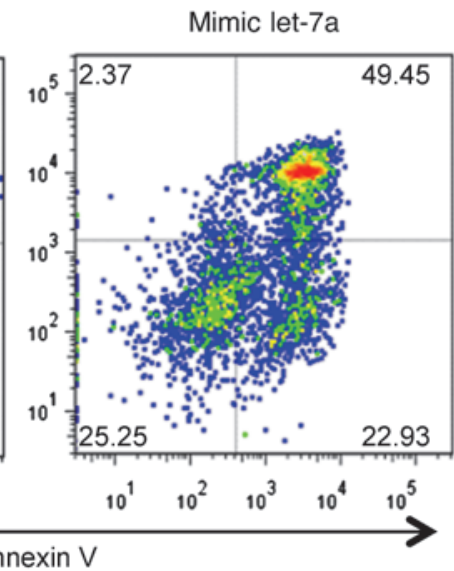

D

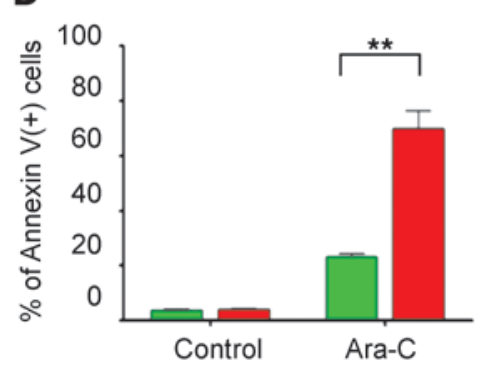

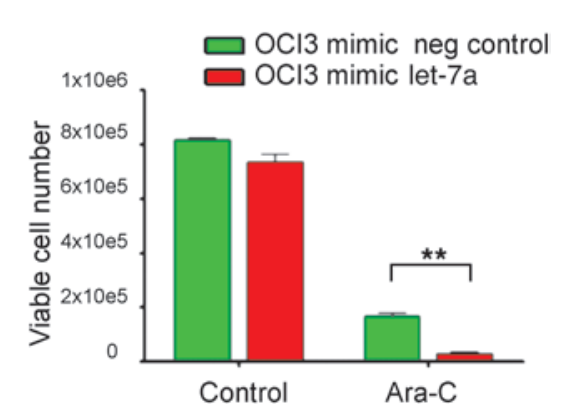

\section{Figure 2}

Transfection of synthetic let-7a sensitizes OCI-AML3 cells to Ara-C treatment. Mimic let-7a or negative control oligonucleotide was transfected into OCl-AML3 cells by electroporation. (A) qRT-PCR showed that cells transfected with mimic had an approximately 3.5-fold increase of let-7a compared with control. (B) Western blots showed cells with mimic let-7a transfection had low expression of let-7a targets such as ITGB3, c-Myc, and BCL-XL. Lane 1, normal culture; lane 2, negative control oligonucleotide; lane 3, mimic let-7a. Expression level relative to normal culture (lane $1)$ is shown below blots. (C) When exposed to Ara-C (2.5 $\mu \mathrm{M}, 48$ hours), the mimic let-7a-transfected cells exhibited $>2$-fold increased apoptosis $\left(69.8 \% \pm 9.4 \%\right.$ vs. $23.2 \% \pm 2.7 \%$ annexin $\left.\mathrm{V}^{+} ; P<0.01\right)$ and significantly lower numbers of viable cells $(27,715 \pm 6025$ vs. $166,878 \pm 12,405$ cells; $P<0.01)$ compared with negative controls. ${ }^{* *} P<0.01$.

tive control oligonucleotide into OCI-AML3 cells by electroporation. Cells were collected 48 hours later for total RNA extraction and Western blotting. qRT-PCR showed that cells transfected with the mimic had an approximately 3.5 -fold increase in let-7a compared with negative controls (Figure 2A). Western blotting indicated that cells with high expression of let-7a had lower expression of ITGB3 (also known as integrin $\beta 3$ ), c-Myc, and BCL-XL (Figure $2 \mathrm{~B}$ ), which suggests that let-7a levels are negatively correlated with the expression of these proteins. When exposed to $2.5 \mu \mathrm{M}$ Ara-C for 48 hours, the let-7a-overexpressing cells exhibited more than 2 -fold increased apoptosis compared with control cells $(69.8 \% \pm$ $9.4 \%$ vs. $23.2 \% \pm 2.7 \%$ annexin $V^{+}$cells; $P<0.01$; Figure $2 \mathrm{C}$ ).

Knockdown of CXCR4 leads to increased let-7a expression in OCI$A M L 3$ cells, which downregulates BCL-XL expression and sensitizes cells to Ara-C. Next, to test whether decreased CXCR4 expression had any functional consequence for cellular chemoresistance, we suppressed CXCR4 in OCI-AML3 cells with lentivirus-delivered CXCR4 shRNA (referred to herein as CXCR4-shRNA-OCI3 cells). Although knockdown of the total CXCR4 level was approximately $30 \%$, as determined by Western blotting, the CXCR4-shRNAOCI3 cells had approximately 70\% lower cell surface CXCR4 expression, as evaluated by flow cytometry (allophycocyaninconjugated anti-CXCR4 antibodies) and displayed approximately $60 \%$ less migration to SDF- $1 \alpha$ compared with the nonsilenc- ing (NS) shRNA control (Figure 3, A and C). Using qRT-PCR, we found that let-7a expression was elevated in CXCR4-shRNAOCI3 cells ( 2.3-fold the level in NS-shRNA-OCI3 cells; Figure 3D). As expected, let-7a-targeted genes such as MYC and BCLXL were substantially decreased in CXCR4-shRNA-OCI3 cells at both the mRNA and the protein level (Figure 3E), inhibiting cell proliferation and increasing sensitivity to Ara-C $(2.5 \mu \mathrm{M}, 64.6 \%$ $\pm 2.4 \%$ vs. $23.6 \% \pm 2.7 \%$ annexin $\mathrm{V}^{+} ; 5 \mu \mathrm{M}, 83.8 \% \pm 4.1 \%$ vs. $44.7 \%$ $\pm 4.3 \%$ annexin $\mathrm{V}^{+} ; P<0.01$ for both; Figure $3, \mathrm{~B}$ and $\left.\mathrm{F}\right)$. Taken together, these results revealed the link between CXCR4 and let$7 \mathrm{a}$ in human AML cells and suggested a novel mechanism for CXCR4-mediated chemoresistance.

let-7a strongly affects chemoresistance of AML cell lines in NOD/ $S C I D / I L-2 r \gamma^{\text {null }}$ mice. We then established 2 AML cell lines, OCIAML3 and Molm13, with stable overexpression of let-7a and luciferase-GFP (referred to herein as OCI3-luc-GFP-let-7a cells and Molm13-luc-GFP-let-7a cells, respectively). Overexpression of let-7a in these cells (confirmed by qRT-PCR) was approximately 1.7-fold in OCI3-luc-GFP-let-7a cells and approximately 1.6fold in Molm13-luc-GFP-let-7a cells relative to specific scrambled control cells (Figure 4A and Figure 5B). In addition, the luciferase signals from the let-7a-overexpressed and scrambled control cells were very similar, as determined by bioluminescence imaging (Figure 4B). let-7a-overexpressing and control cells were 
A

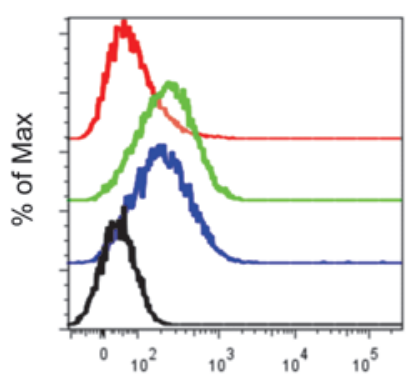

B

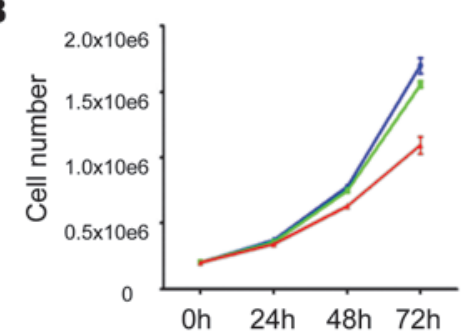

D

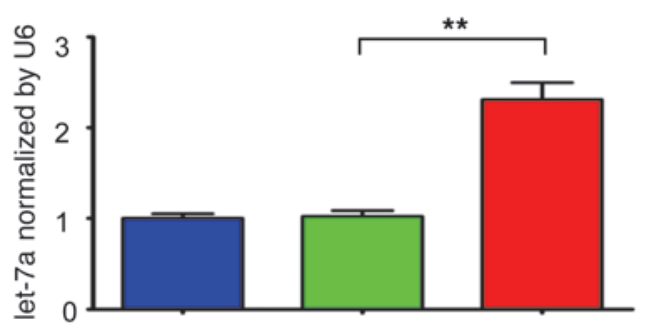

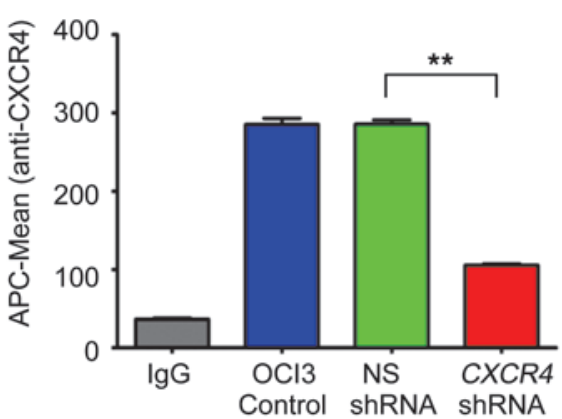

C

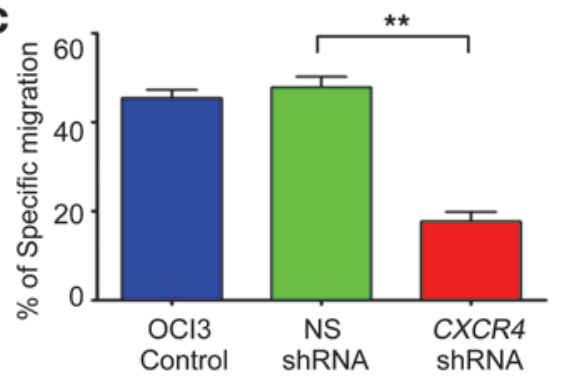

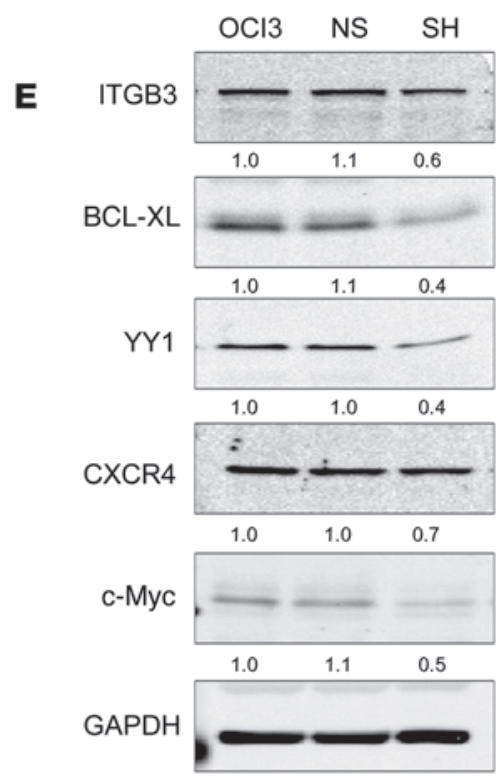

$\mathbf{F}$

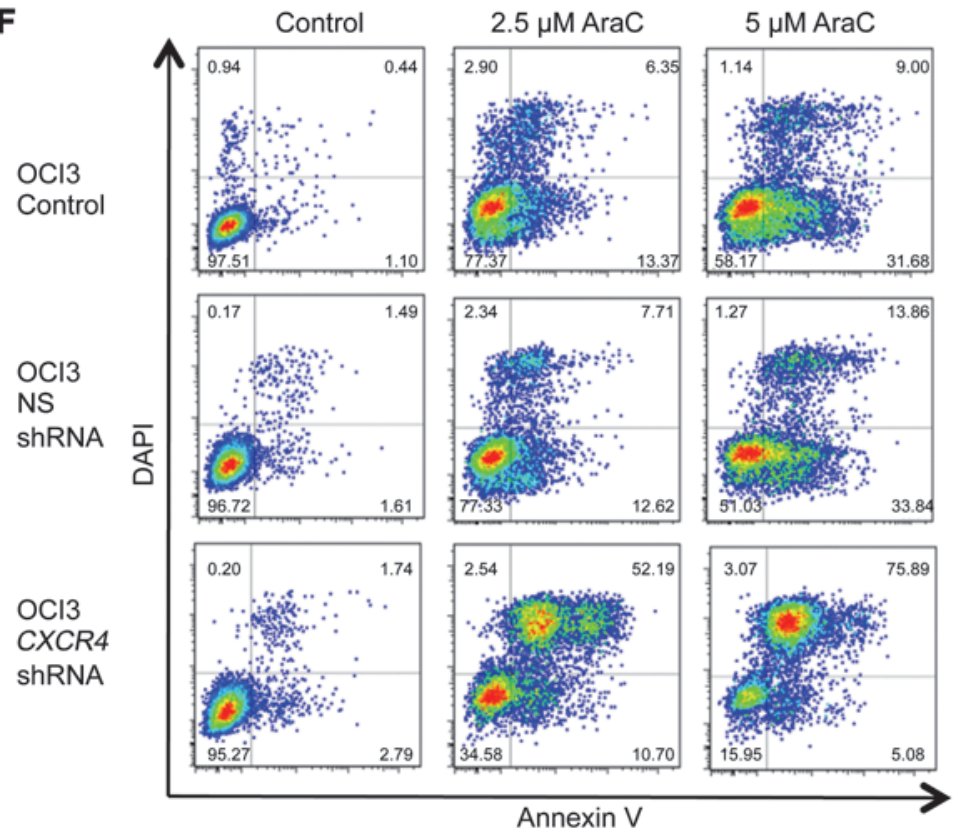

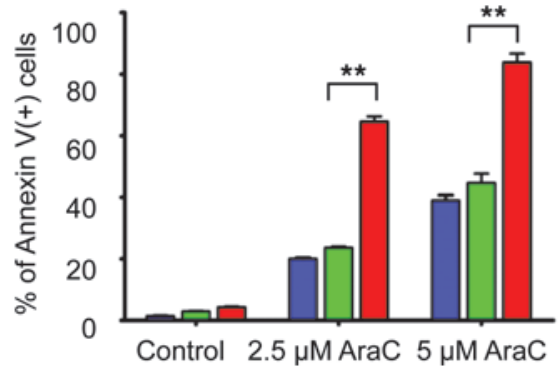

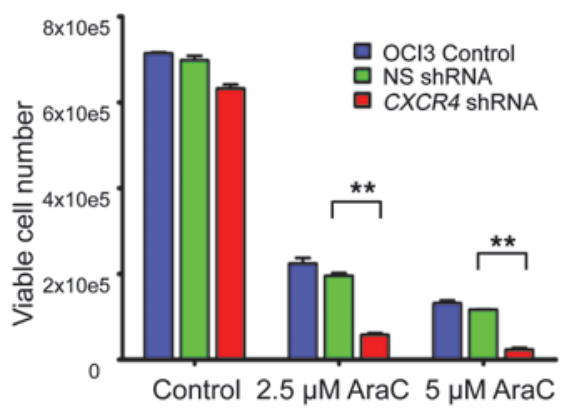

Figure 3

Knockdown of CXCR4 leads to upregulation of let-7a expression in OCI-AML3 cells. CXCR4 was suppressed in OCI-AML3 cells with lentivirusdelivered CXCR4 shRNA. (A) The resulting CXCR4-shRNA-OCI3 cells had approximately $70 \%$ lower cell surface CXCR4 expression, as evaluated by flow cytometry. (B) CXCR4-shRNA-OCI3 cells grew slower than parental control and NS-shRNA-OCI3 cells. (C) In addition, approximately $60 \%$ less migration to SDF-1 $\alpha$ was observed for CXCR4-shRNA-OCI3 cells. (D) qRT-PCR showed that let-7a was elevated in CXCR4-shRNA$\mathrm{OCI} 3$ cells ( $\sim 2.3$-fold the level in NS-shRNA-OCI3 cells). (E) As expected, let-7a-targeted genes such as MYC and BCLXL were decreased in CXCR4-shRNA-OCI3 cells. Expression level relative to parental OCI-AML3 cells is shown below blots. (F) CXCR4-shRNA-OCI3 cells exhibited significantly higher sensitivity to Ara-C, as shown by apoptosis rates and absolute viable cell counts. ${ }^{* *} P<0.01$. 
A

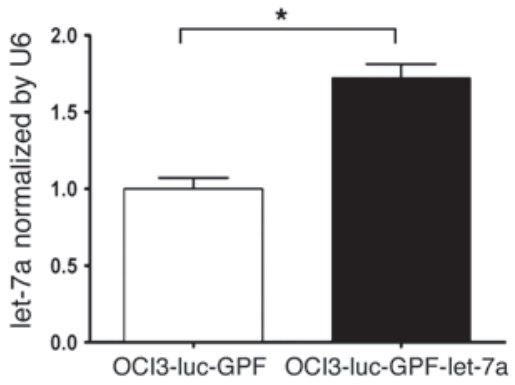

C
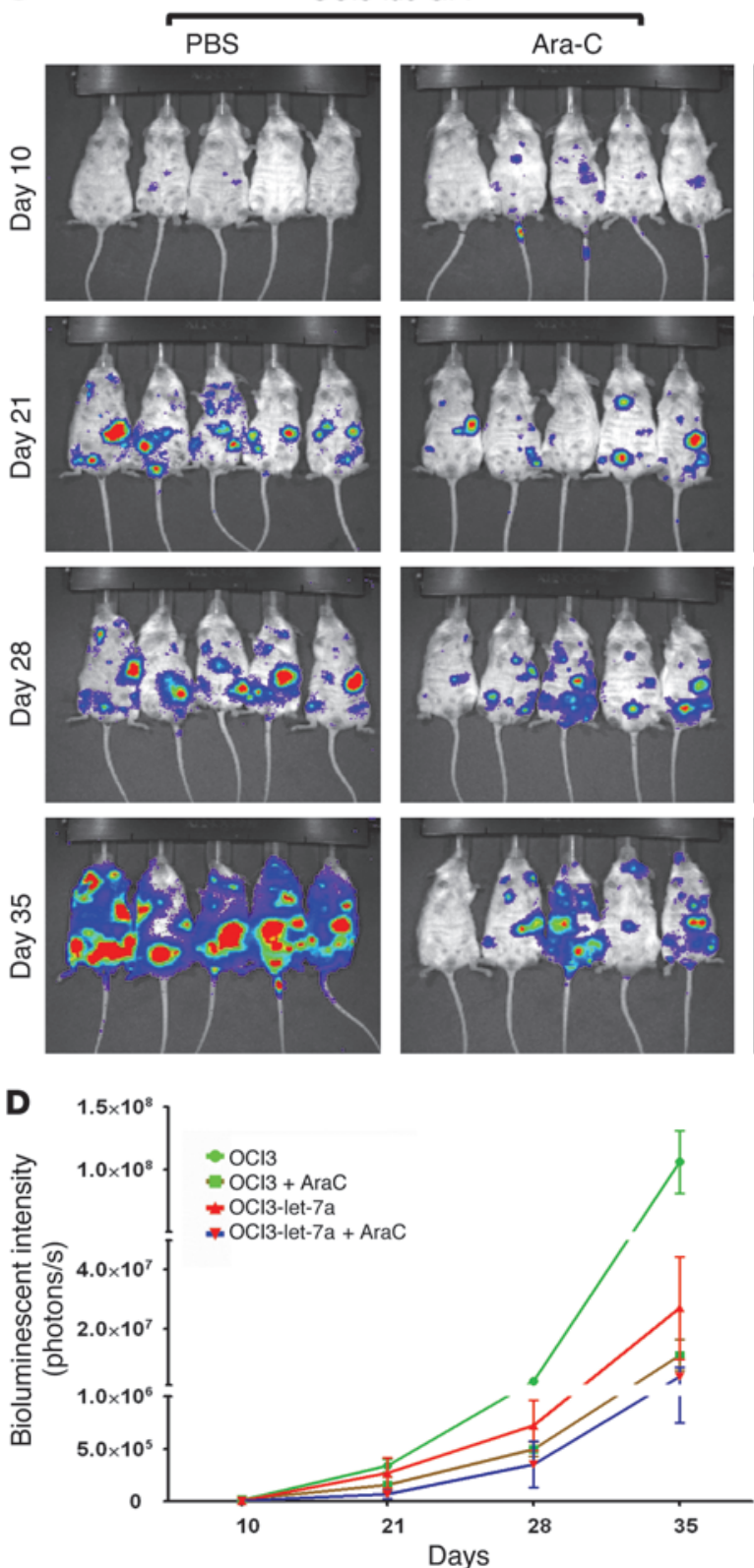

B

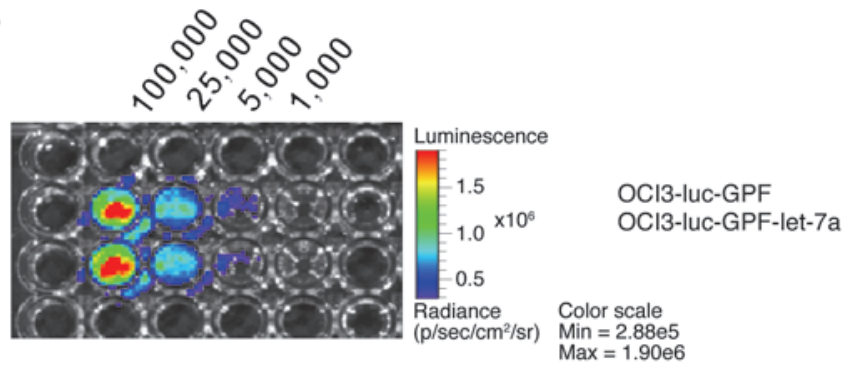

Figure 4
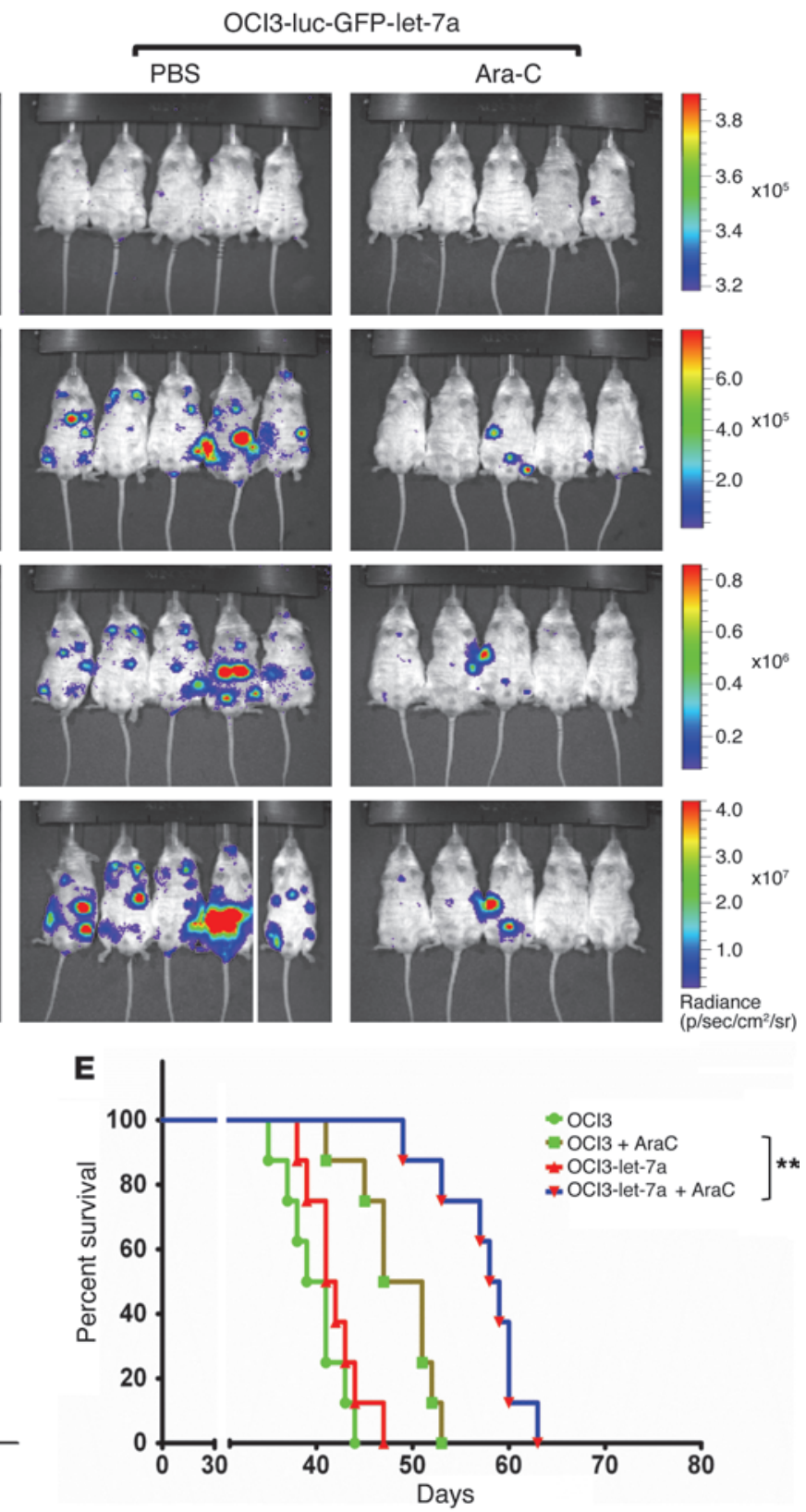

let-7a strongly affects chemoresistance of OCI-AML3 cells in NSG mice. (A) OCl3-luc-GFP-let-7a cells were established by lentiviral infection, and let-7a overexpression was confirmed by qRT-PCR. (B) Luciferase signals were similar between OCI3-luc-GFP-let-7a and control cells, as determined by bioluminescence imaging. (C) OCl3-luc-GFP-let-7a and control cells were transplanted into NSG mice $(n=8)$ for leukemia development, and mice were treated with PBS or Ara-C twice weekly from day 7 after cell injection. On days 10,21, 28, and 35, mice were imaged after D-luciferin injection. Serial images of 5 representative mice at different time points are shown. (D) Bioluminescence imaging was quantified and expressed as luminescent intensity. (E) Overall survival rate in each group was estimated by Kaplan-Meier method. ${ }^{\star} P<0.05,{ }^{\star \star} P<0.01$. 
A

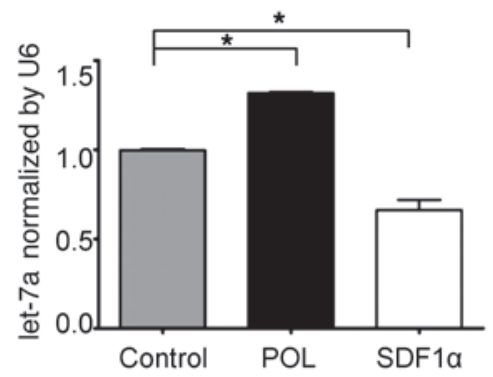

B

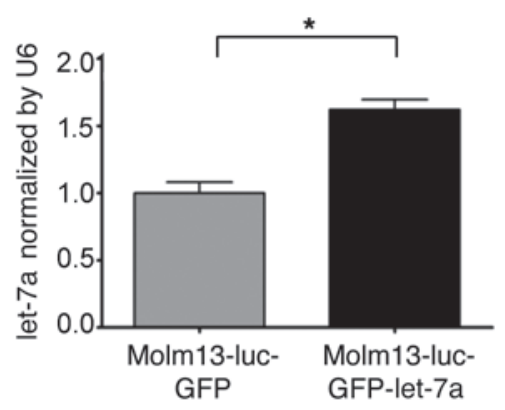

C

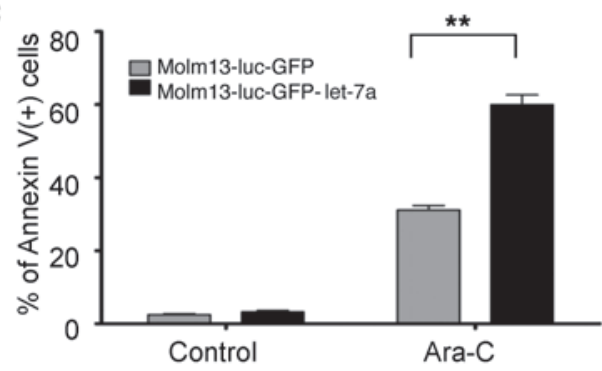

D

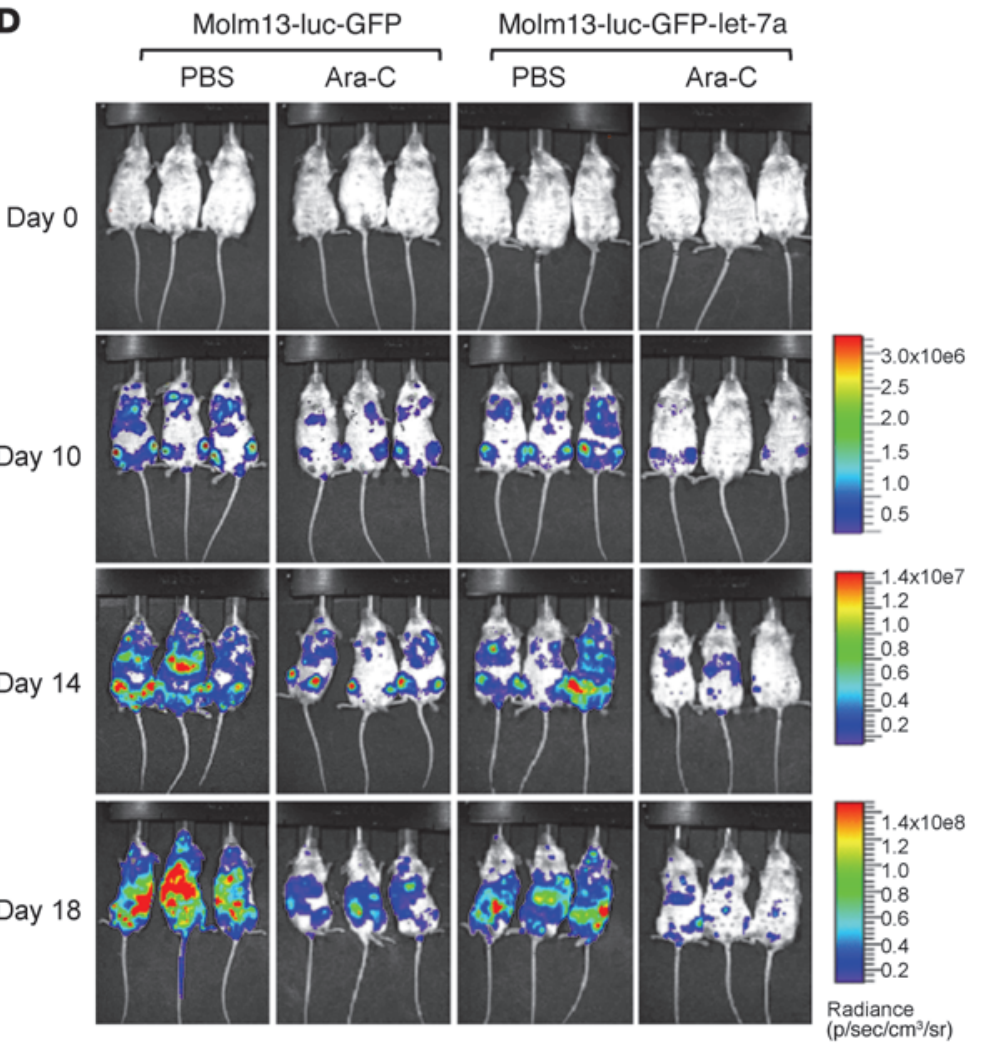

E
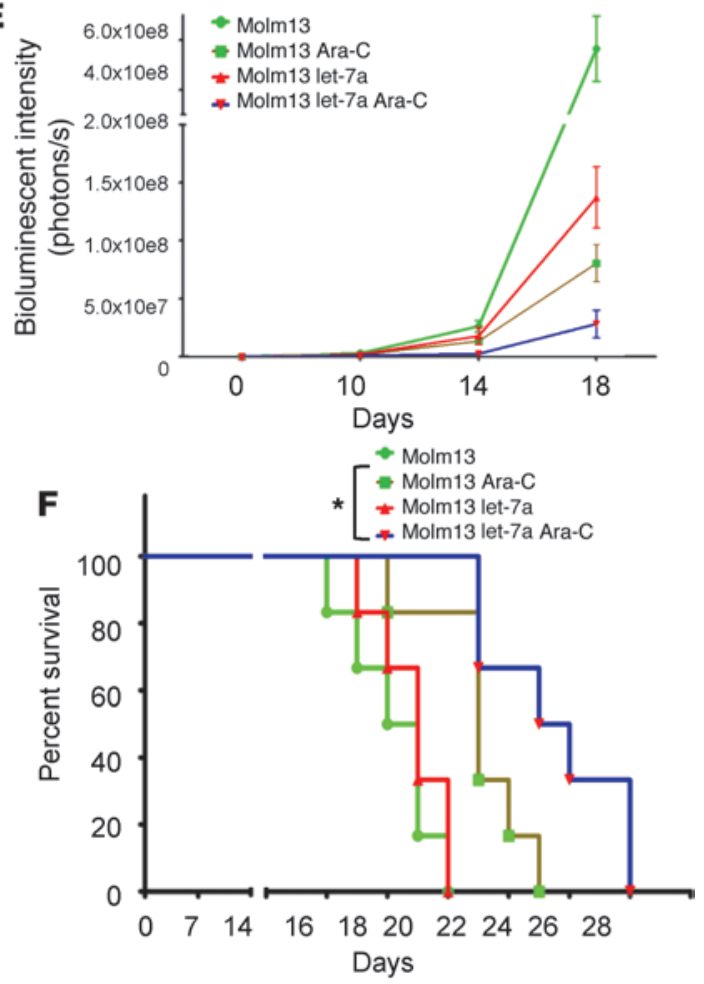

G
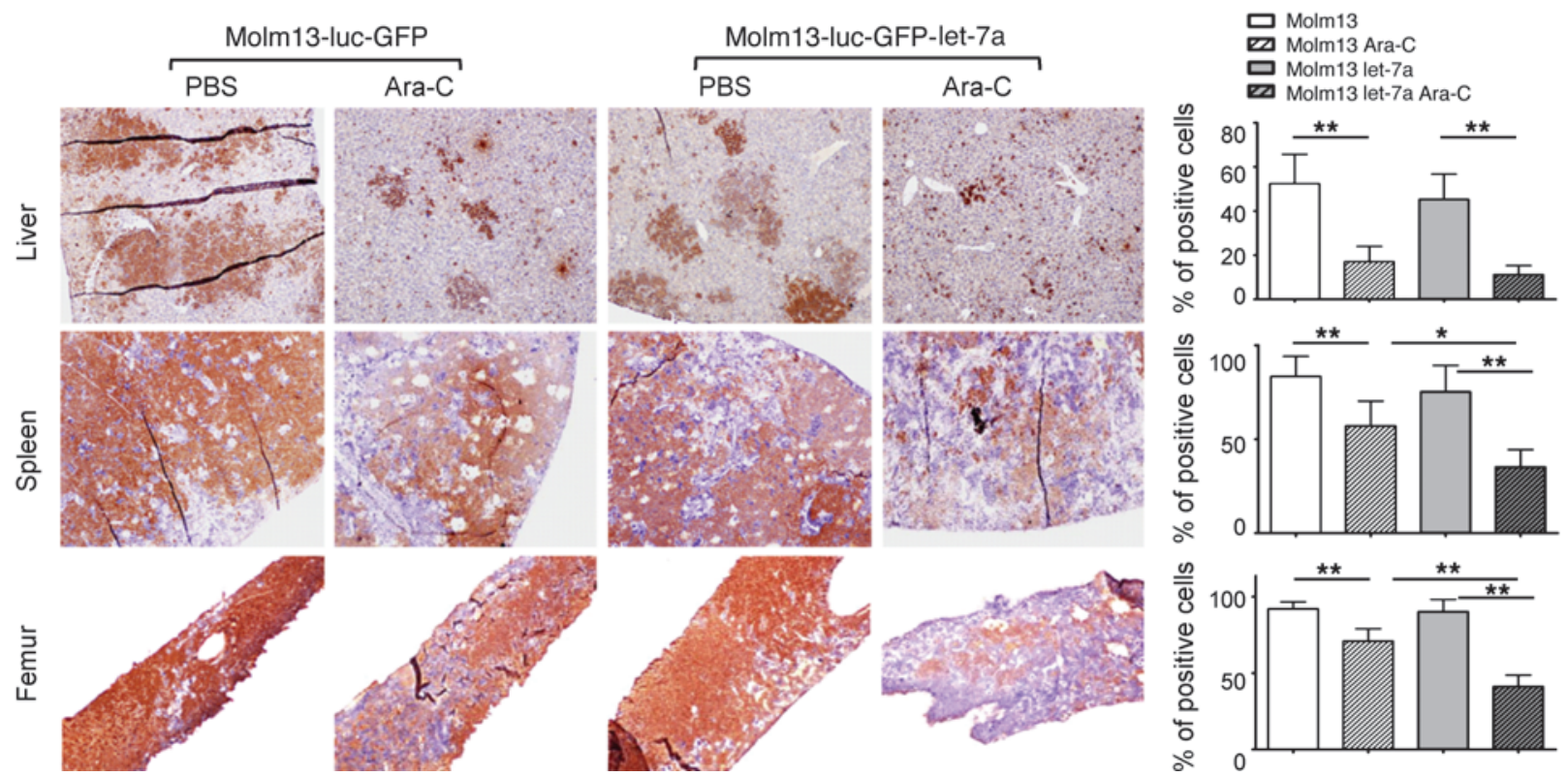


\section{Figure 5}

Molm13 cells with overexpression of let-7a are more sensitive to chemotherapy in vitro and in vivo. (A) Expression of let-7a in Molm13 cells was upregulated with POL6326 treatment and decreased with SDF- $1 \alpha$ administration, as determined by qRT-PCR. (B) Molm13luc-GFP-let-7a cells were established by lentiviral infection, which showed approximately 1.6-fold more let-7a compared with the scrambled control. (C) Molm13-luc-GFP-let-7a cells exhibited significantly higher sensitivity to Ara-C treatment in vitro. (D) Molm13-luc-GFPlet-7a and scrambled control cells were injected into NSG mice $(n=$ 8 ), and the mice were treated with either PBS or Ara-C twice a week from day 7 . Serial images of 3 representative mice on days $0,10,14$, and 18 after D-luciferin injection are shown. (E) Bioluminescence imaging was quantified in terms of luminescent intensity. $(\mathbf{F})$ Overall survival rate in each group was estimated by Kaplan-Meier method $\left({ }^{\star} P<0.05\right)$. (G) 3 representative mice per group were sacrificed on day 18 , and tissues were fixed and sliced for immunohistochemistry staining with an anti-firefly antibody to specifically identify human leukemic cells. Analysis of spectral images further confirmed the significantly reduced leukemia burden in Ara-C-treated Molm13-lucGFP-let-7a cells. Original magnification, $\times 20$ (liver and spleen); $\times 4$ (femur). ${ }^{*} P<0.05,{ }^{* *} P<0.01$.

then transplanted into NOD/SCID/IL-2rynull (NSG) mice for leukemia development $(n=8)$, and the mice were treated with either PBS or Ara-C twice a week beginning at day 7 after cell injection. Bioluminescence imaging was used to monitor the tumor burden at different time points. Mice with OCI3-luc-GFP-let-7a cells showed less luciferase activity and minimally extended survival compared with mice injected with control cells (median survival duration, 41.5 vs. 40.0 days; $P=0.2$; Figure 4 , C and D). Ara-C treatment reduced the tumor burden and improved survival, but the median survival was significantly longer in the OCI3-lucGFP-let-7a group than the control group (58.5 vs. 49.0 days; $P<$ 0.01; Figure 4E). Similarly, in Molm 13 mice with Ara-C administration, those injected with Molm13-luc-GFP-let-7a cells lived longer than those injected with control cells $(P<0.05$; Figure 5, D-F). Moreover, representative mice were sacrificed on day 18 , and tissues such as liver, spleen, and bone (femur) were fixed and sliced for immunohistochemistry staining with an anti-firefly antibody. Analysis of spectral imaging (see Methods) further confirmed that Ara-C treatment reduced the leukemia burden, and the effect was more obvious in the mouse group bearing Molm13-luc-GFP-let-7a cells (Figure 5G). Taken together, these data suggested that let-7a overexpression sensitized AML cells to Ara-C treatment in vivo.

CXCR4 regulates let-7a expression via YY1. To further explore the link between let-7a and CXCR4, we treated OCI-AML3 cells with SDF-1 $\alpha$ or CXCR4 antagonist and performed qRT-PCR for primary LET7A1 (pri-let-7a-1), pri-let-7a-2, and pri-let-7a-3. All 3 primary miRNAs were downregulated by SDF- $1 \alpha$ and upregulated by POL6326 (Figure 6A), which indicated that let-7a was regulated at the transcriptional level in this CXCR4-mediated signaling. We then retrieved the promoter sequences for each gene $(5,000 \mathrm{bp}$ upstream of the initiation transcription site) and exhaustively searched for all potential binding sites using ALGGEN-PROMO (24). The search site predicted a series of transcription factors that might be involved in the transcriptional regulation of let-7a (Table 1). The transcription factor Yin Yang 1 (YY1), ranked first in the prediction, is a member of the polycomb group and has been shown to be involved in CXCR4 signaling (25-29).
qRT-PCR, Western blotting, and immunocytochemistry showed upregulation of YY1 mRNA by SDF-1 $\alpha$ treatment and its downregulation with exposure to CXCR4 antagonist (Figure 6B), which suggests that YY1 was involved in CXCR4 signaling in AML cells. To test whether YY1 is associated with let-7a expression, ChIP assay were performed, and the results confirmed the interaction between YY1 and pri-let-7a-1, pri-let-7a-2, and pri-let-7a-3 DNA fragments. The association was enhanced by SDF-1 $\alpha$ treatment, which was not detected by normal IgG (Figure 6C). It has been reported that YY1 transcriptionally represses miR-29b/c through recruitment of the polycomb complex and the resultant trimethylation of H3K27 (30). We asked whether a similar regulation occurred on the let-7a locus. In order to prove this hypothesis, YY1 expression in OCI-AML3 cells was knocked down by specific YY1 shRNA. The resulting YY1-shRNA-OCI3 cells showed a 3-fold increase of let-7a expression and were much more sensitive to Ara-C treatment than were control cells (Figure 6D). ChIP analysis was performed on chromatin obtained from cells transfected with control or YY1 shRNA. As expected, shRNA-mediated reduction of YY1 resulted in loss of H3K27 methylation at promoter regions of pri-let-7a-1, pri-let-7a-2, and pri-let-7a-3 (Figure 6E). Together, these data revealed that YY1 levels were inversely correlated with let-7a levels in OCI-AML3 cells, which suggests that CXCR4 mediates let-7a regulation at least partially through YY1.

Lower let-7a levels in AML patient samples are associated with higher CXCR4 expression and chemoresistance. To determine the pathologic relevance of the relationship between CXCR4 and let-7a, we analyzed surface CXCR4 and let-7a expression levels in $50 \mathrm{AML}$ patient samples. The ratio of anti-CXCR4 antibody MFI to IgG MFI was determined, and the samples were categorized into 2 groups according to their MFI ratios ( $<5$ or $>5 ; n=25$ per group). CXCR4 surface expression was inversely associated with let-7a levels: the samples with lower CXCR4 MFI ratios had significantly higher let-7a expression than those with higher ratios $(P<0.001$; $r^{2}=0.53$; Figure 7A).

Because CXCR4 receptors in human AML samples are heterogeneous, we stained 10 fresh samples with CXCR4 antibody and sorted the cells as CXCR $4^{\text {hi }}$ or CXCR $4^{\text {lo }}$ (gated on the $30 \%$ highest and the 30\% lowest CXCR4 staining cells). The CXCR4hi subpopulation expressed substantially lower levels of let-7a (Figure 7B). ITGB3 and BCL-XL proteins were expressed at much lower levels in the $\mathrm{CXCR}^{\mathrm{lo}}$ subpopulation (Figure 7C), and the CXCR4 ${ }^{\text {lo }}$ subpopulation was more sensitive to Ara-C treatment (Figure 7D), which may explain why low surface CXCR4 expression in AML cells was correlated with better prognosis, resulting in longer relapse-free and overall survival times.

Next, primary human AML cells stably overexpressing let-7a were established for xenograft models. A 2.2-fold increase of let-7a was achieved after the virus infection (Figure 7E). 15 days after cell injection, human $\mathrm{CD}_{4} 5^{+}\left(\mathrm{hCD} 45^{+}\right)$cells were observed in the peripheral blood of all mice (range, $0.5 \%-1.2 \%$ ). As determined by peripheral blood hCD $45^{+}$cell percentages, leukemia burden was markedly reduced with Ara-C treatment, an effect that was more pronounced in the let-7a-overexpressing group (Figure 7F). Next, 3 representative mice per group were sacrificed on day 32 , and the spleens were isolated, weighed, and fixed for sectioning. The spleen size of the Ara-C-treated let-7a-overexpressing group was significantly smaller than the other groups, and immunohistochemical staining with anti-hCD45 antibody confirmed a lower leukemia burden (Figure $7 \mathrm{G})$. Finally, we observed the median survival to be significantly 
A
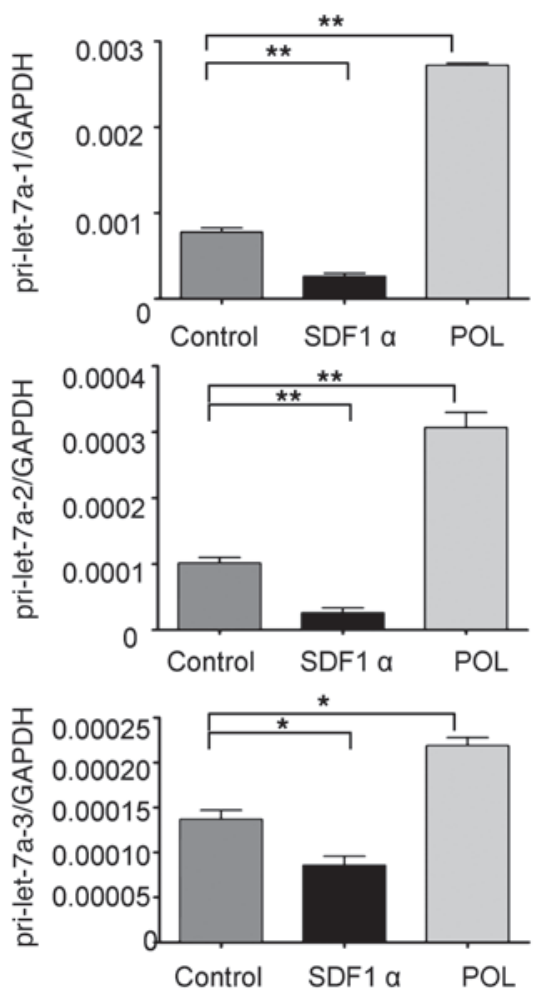

C
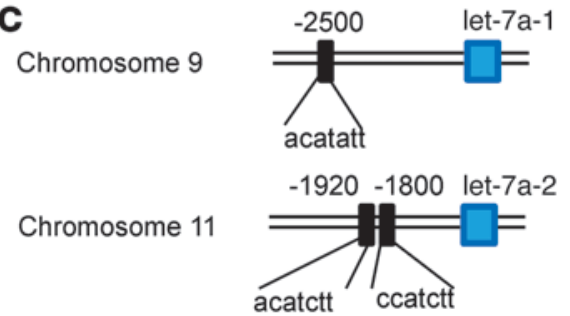

Chromosome 22

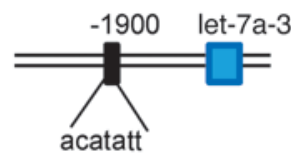

B
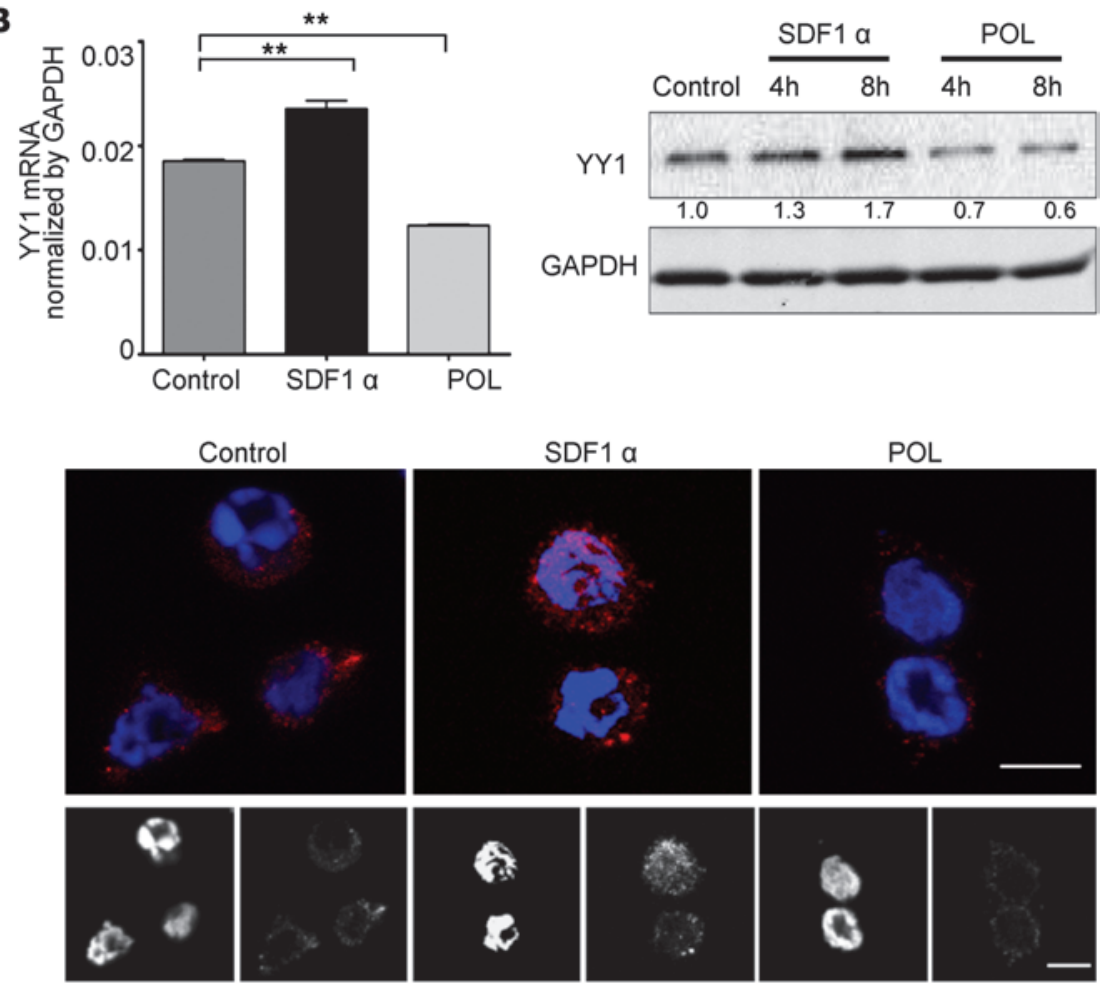
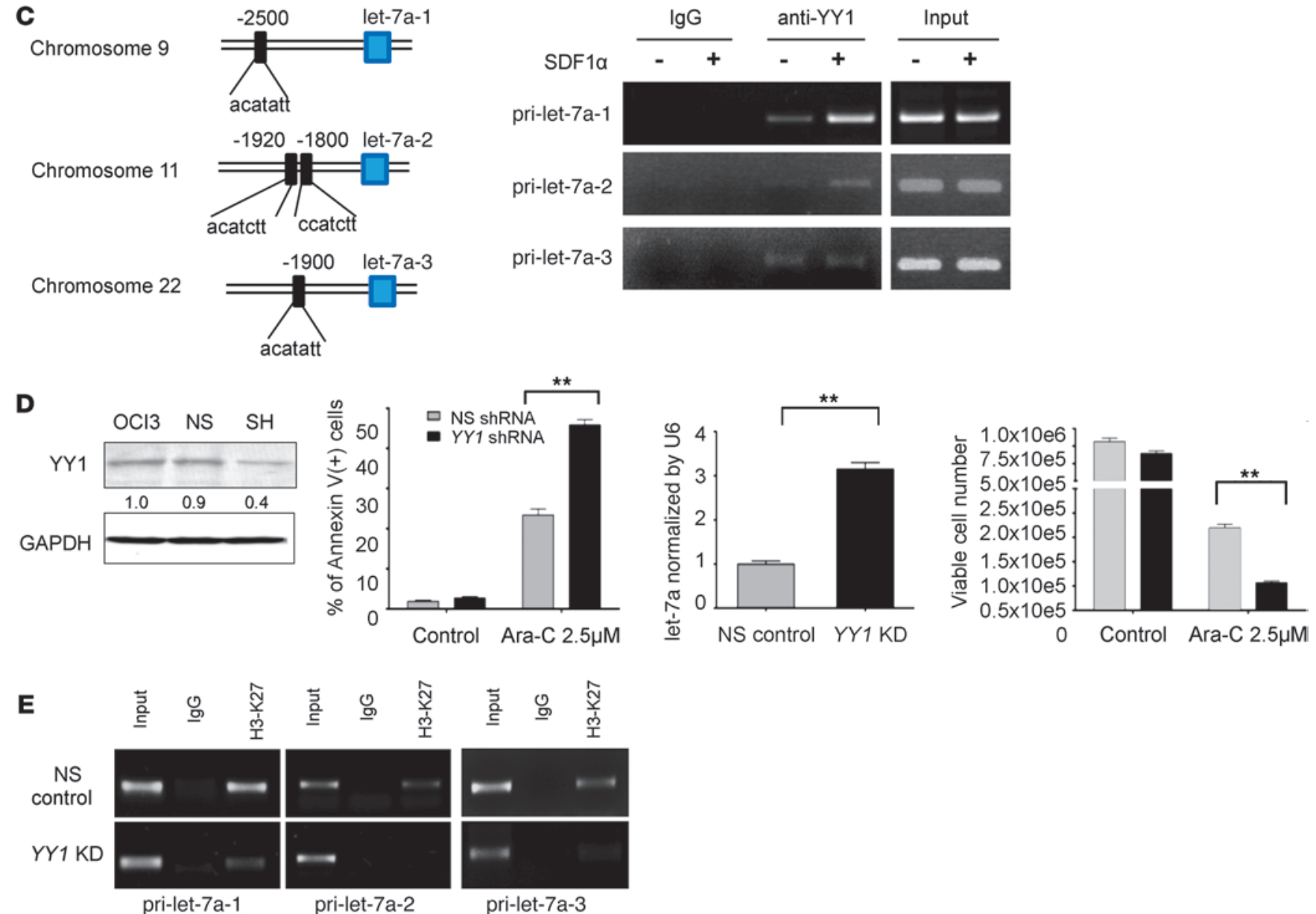


\section{Figure 6}

let-7a expression regulated by CXCR4 signaling is partially mediated though YY1. (A) qRT-PCR demonstrated that pri-let-7a-1, pri-let-7a-2, and pri-let-7a-3 were downregulated by SDF-1 $\alpha$ and upregulated with POL6326 treatment. (B) qRT-PCR, Western blotting, and immunocytochemistry confirmed the upregulation of $Y Y 1$ with SDF- $1 \alpha$ treatment and its reduction by CXCR4 antagonist at the mRNA and protein levels. Expression level relative to control is shown below blots. Scale bars: $10 \mu \mathrm{m}$. (C) Specific primers were designed for let-7a DNA fragments that include the YY1 binding sites. ChIP assay further confirmed that the interaction between $Y Y 1$ and the pri-let-7a-1, pri-let-7a-2, and pri-let-7a-3 DNA fragments was enhanced by SDF-1 $\alpha$ treatment. (D) YY1 expression in OCl-AML3 cells was knocked down by shRNA, and the cells showed a significantly higher level of let-7a compared with NS-shRNA-OCI3 cells. Moreover, YY1-shRNA-OCI3 cells were more sensitive to Ara- $C$ treatment. Expression level relative to parental $\mathrm{OCI}-$ AML3 cells is shown below blots. (E) ChIP assays were performed with control IgG or antibody to H3K27 on the chromatin obtained from cells transfected with either control or YY1 shRNA. The immunoprecipitated chromatin was analyzed by PCR using specific primers (see Methods). ${ }^{\star} P<0.05,{ }^{* *} P<0.01$.

extended in the let-7a-overexpressing versus the control group under Ara-C treatment: $80 \%$ with let-7a-overexpressing AML were still alive on day 75 (leukemia free by human CD45 flow cytometric analysis), compared with $0 \%$ of controls $(P<0.01$; Figure $7 \mathrm{H})$.

\section{Discussion}

There is accumulating evidence that dysregulation of miRNA expression is involved in various cancers, including hematological malignancies (31-34). Here, we identified for the first time a CXCR4/let-7a/BCL-XL axis in human AML cells that may be responsible for the chemoresistance observed in the clinical setting. In the present study, we showed that overexpression of surface CXCR4 and activation of CXCR4 signaling resulted in downregulation of let-7a, which led to enhanced expression of the antiapoptotic protein BCL-XL in AML cells. Collectively, our results suggest that let-7a plays an important role in AML cell chemoresistance and are indicative of a new mechanism by which miRNA is involved in the SDF-1 $\alpha /$ CXCR4 signaling pathway.

Over the last few years, CXCR4 surface expression has been reported as an independent prognostic factor for disease relapse and survival in AML patients $(3,35,36)$. Our group and others previously demonstrated that targeting the SDF- $1 \alpha /$ CXCR 4 axis by CXCR 4 inhibition can ameliorate resistance of AML cells to chemotherapy in vitro, in vivo, and in clinical trials $(8,9,37)$. To further explore the mechanism of targeting CXCR4, we evaluated the expression of miRNAs, which have been reported to be differentially expressed and deregulated in pathophysiological conditions such as cancers. We identified a series of miRNAs that were altered by SDF-1 $\alpha$ / CXCR4 modulation. let-7a was significantly downregulated in OCIAML3 cells after SDF- $1 \alpha$ treatment and highly elevated by treatment with CXCR4 antagonists. let-7, the second miRNA ever identified, mainly controls developmental timing in Caenorhabditis elegans and is evolutionary conserved across species $(38,39)$. Recent studies have shown that let-7 is involved in various biological processes and likely as a tumor suppressor (40). In addition, it was recently reported that the antiapoptotic protein BCL-XL is also a target of let-7a in human hepatocellular carcinoma (41). This information led to our hypothesis that let-7a is responsible for CXCR4 signaling-mediated chemoresistance in AML cells.
Consistent with our hypothesis, when we suppressed CXCR4 in AML cell lines with lentivirus-delivered CXCR4 shRNA, the cells showed significantly higher let-7a levels than did scrambled controls. In our study, knockdown of CXCR4 expression or transfection of a low amount of let-7a into AML cells was not capable of inducing apoptosis, despite a clear reduction in BCL-XL, but these approaches substantially enhanced sensitivity to Ara-C both in vitro and in vivo. Expression of ITGB3, a protein strongly related to cell adhesion and migration, was also repressed by let-7a in AML cells. There are more than 5,000 other potential targets of let-7a predicted by microRNA.org, based on the miRanda algorithm. Genes including RAS, HMGA2, MYC, IL6, MUC4, CDC25A, $C A S P 3$, and $C D K 5$, which are involved in tumorigenesis, cell cycle and apoptosis regulation, and posttranscriptional control of immune responses, have been reported to be targets of let-7a in different studies (42-46). However, it may not be straightforward to assign miRNA function in AML cells based on function in other cell types, as the same miRNA can have distinct biological activities in different cellular contexts (47). Although our present findings established a link between CXCR4 and let-7a, detailed studies to determine more targets of let-7a may further elaborate the mechanism of CXCR4 signaling-mediated chemoresistance in AML. In further exploring the links between let-7a and CXCR4, we found that let-7a was regulated at a transcriptional level in OCI-AML3 cells treated with either SDF-1 $\alpha$ or CXCR4 antagonist. Preliminary analysis of the promoter region of let-7a suggested that a series of transcription factors may be involved in the transcriptional regulation of the miRNA. The transcription factor YY1, which functions to silence transcription of a selected set of genes by chromatin modification, was demonstrated to play a role in CXCR4-mediated let-7a regulation.

We also found that let-7a levels in primary human samples were inversely correlated with CXCR4 surface expression, and the CXCR4 surface expression of primary samples was heterogeneous in different

\section{Table 1}

PROMO "MultiSearchSites" output for regulation regions of prilet-7a-1, pri-let-7a-2, and pri-let-7a-3

\begin{tabular}{lc} 
Rank & Transcription factor \\
1 & YY1 \\
2 & GR- $\alpha$ \\
3 & C/EBP $\beta$ \\
4 & GR- $\beta$ \\
5 & TFIID \\
6 & GATA-1 \\
7 & TFII-I \\
8 & AP-2 $\alpha$ A \\
9 & GR \\
10 & HNF-3 $\alpha$ \\
11 & RXR- $\alpha$ \\
12 & p53 \\
13 & C-Ets-1 \\
14 & FOXP3 \\
15 & ER- $\alpha$ \\
16 & IRF-2 \\
17 & XBP-1 \\
18 & Pax-5 \\
& \\
\hline
\end{tabular}

Transcription factors predicted within a dissimilarity margin $\geq 1 \%$. Only those binding site predictions that appear in all 3 sequences are shown. 
A

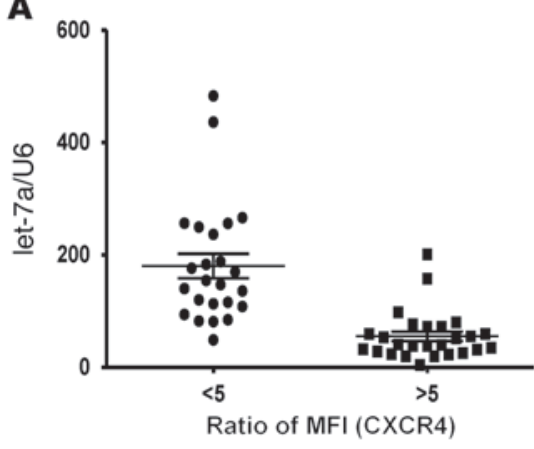

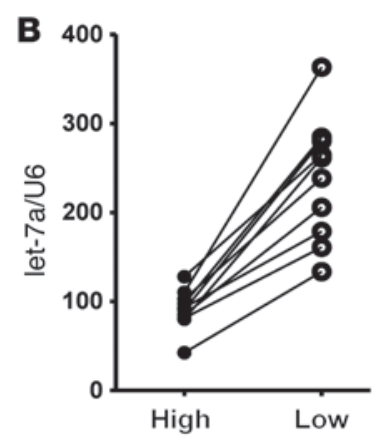

D

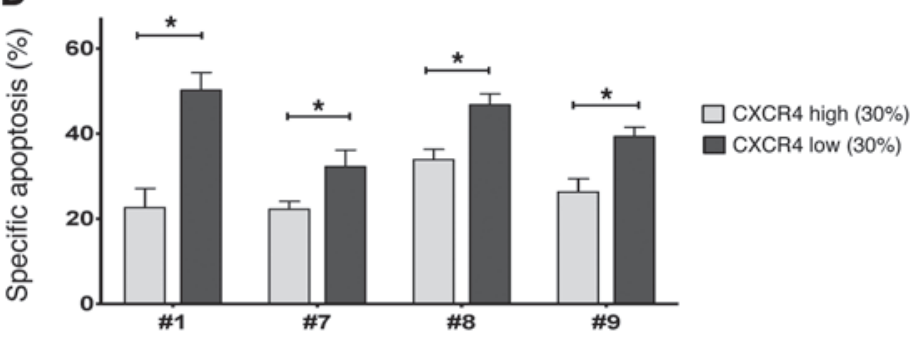

ODay 15
$\mathbf{F}$

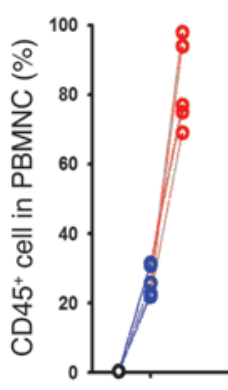

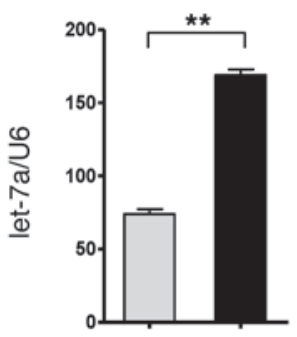

EV let-7a

- Day 24

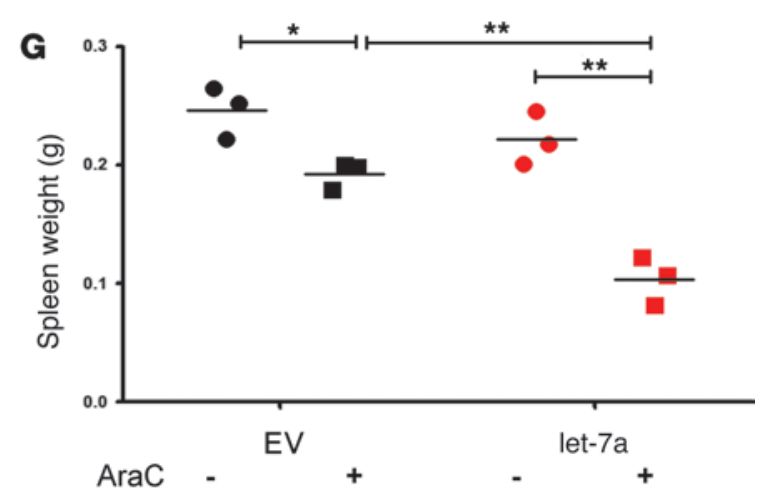

EV

AraC - +
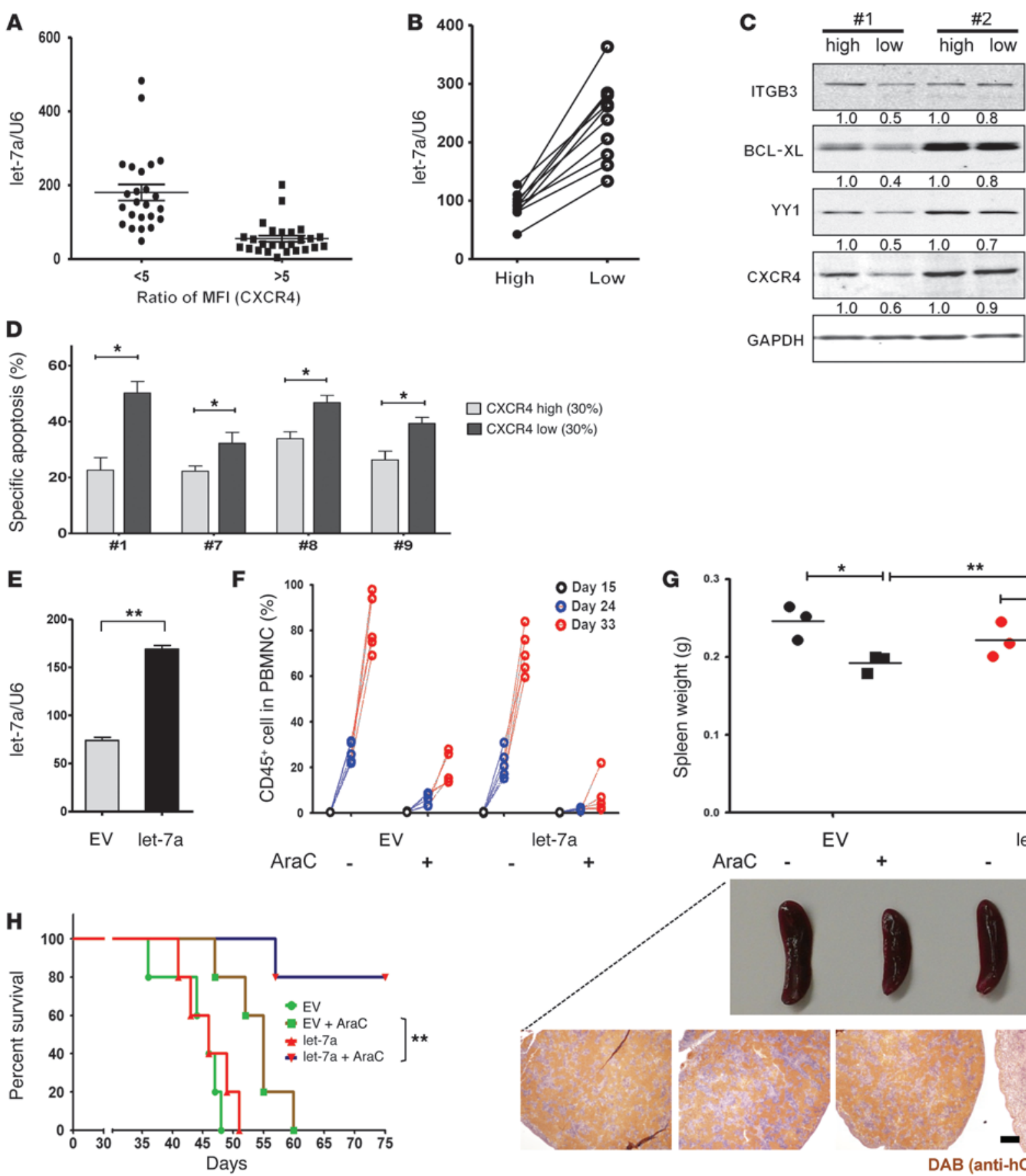

AraC
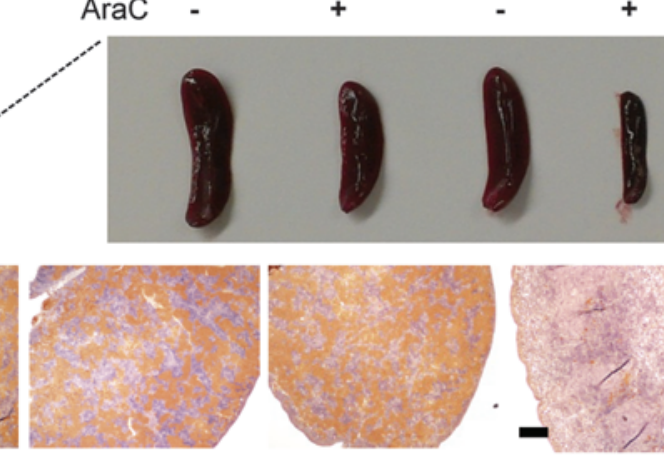

Figure 7

let-7a expression inversely correlates with surface CXCR4 expression and chemoresistance in AML patient samples. (A) 50 AML patient samples were divided according to MFI ratio (anti-CXCR4 MFI/lgG MFI). Samples with higher MFI ratios ( $>5$ ) had significantly higher let-7a expression than those with lower MFI ratios $(<5)(P<0.001 ; n=25$ per group). (B) 10 fresh AML samples were stained with CXCR4 antibody and sorted based on surface CXCR4 expression. The CXCR4 $4^{\text {hi }}$ subpopulation showed substantially less let-7a. (C) Western blot of samples 1 and 2 showed that let-7a targets, such as ITGB3 and BCL-XL, were expressed much less in the CXCR4 ${ }^{\text {lo }}$ subpopulation. Expression level relative to CXCR4hi cells is shown below blots. (D) The CXCR4 $4^{\text {lo }}$ subpopulation was more sensitive to Ara-C treatment. Specific apoptosis was calculated as (percent treatment apoptosis - percent spontaneous apoptosis)/(1 - percent spontaneous apoptosis) and expressed as a percentage. Results for samples 1, 7, 8, and 9 are shown. (E) Primary human AML cells were stably infected with let-7a or empty vector (EV) and expanded for xenograft models, which showed a 2.2-fold increase in let-7a versus control cells. (F) hCD45 ${ }^{+}$cells in mouse peripheral blood were determined using flow cytometry at different time points. $\mathrm{hCD} 45^{+}$cell percentage was significantly reduced with Ara- $C$ treatment, which was more obvious in the let-7a-overexpressing group. (G) 3 representative mice per group were sacrificed on day 32. Spleens of the Ara-C-treated let-7a-overexpressing group were significantly smaller than the other groups, and immunohistochemical staining with anti-hCD45 antibody (original magnification, $\times 4$ ) confirmed lower leukemia burden. Scale bar: $100 \mu \mathrm{m}$. (H) Median survival was significantly extended in the let-7a-overexpressing group compared with controls with Ara-C treatment. ${ }^{*} P<0.05,{ }^{* *} P<0.01$. 
Table 2

Characteristics of AML patients with high and low CXCR4 MFI ratios

\section{Characteristic}

CXCR4 MFI ratio

$$
>5(n=25)
$$

$<5(n=25)$

Sex

\section{Male}

Female

FAB subtype

MO

M1

M2

M3

M4

M5

$\begin{array}{cc}11(44 \%) & 13(52 \%) \\ 14(56 \%) & 12(48 \%) \\ 3(12 \%) & 10(40 \%) \\ 5(20 \%) & 3(12 \%) \\ 5(20 \%) & 8(32 \%) \\ 0(0 \%) & 1(4 \%) \\ 11(44 \%) & 3(12 \%) \\ 1(4 \%) & 0(0 \%)\end{array}$

Values are expressed as $n$ (\%). FAB, French-American-British classification.

French-American-British classification subtypes: $44 \%$ of the cases with high CXCR4 MFI ratios in this study were M4, and $40 \%$ of cases with low CXCR4 MFI ratios were M0 (Table 2). This finding is consistent with other published studies showing that CXCR4 surface expression is higher in M4/M5 (myelomonocytic) and M3 (promyelocytic) AMLs, and lower in M0 (minimally differentiated), M1/M2 (myeloid), and M6 (erythroid) AMLs (48). High blast percentages and high levels of serum LDH were observed in CXCR4-high patients (Table 3); however, $P$ values were not significant, possibly due to the limited number of cases analyzed. A significant difference with respect to wbc number, which is also a negative prognostic marker in AML, was noted between the CXCR4-high and -low cases; significant negative correlations between CXCR4 and percentage of CD117 and CD34 expression were also detected (Table 3 ). It was previously reported that the number of $\mathrm{CD} 34^{+}$blasts in bone marrow was dramatically reduced by administration of the CXCR4 inhibitor AMD3100 (49). The c-Kit receptor CD117, which is considered to play a crucial role in hematopoiesis, has also been shown to be involved in the regulation of leukemia blast egress into the peripheral blood (50). The observation of a low percentage of circulating $\mathrm{CD} 117^{+}$and $\mathrm{CD} 34^{+}$leukemic blasts suggests that CXCR4 is essential for retention of these leukemic populations in the marrow. In our study, we linked the regulation of CXCR4 to the amount of let-7a present in AML cells. In addition, let7 a was shown to be a tumor suppressor in that it repressed expression of BCL-XL, which is strongly involved in leukemia chemoresistance. This finding assigns possible therapeutic relevance to let-7a, as we were able to show that the sensitivity of AML cells to chemotherapy was strongly enhanced when cells were transfected with pri-let-7a. Restoration of let-7a to its normal expression level could be an attractive and promising novel concept in AML therapy; however, developing a technique for safe and efficient delivery and in situ administration is still a major obstacle. Efficiently targeting CXCR4 would be the best alternative at the current stage. Together, our results extend the concept of targeting CXCR4 in AML treatment by defining a complex network with the miRNA let-7a.

\section{Methods}

Mice. NSG mice were purchased from Jackson Laboratory.

Cell lines, primary samples, and cultures. Human AML cell lines OCI-AML3 and Molm13 were cultured in RPMI 1640 medium supplemented with $10 \%$ FBS. Cells were transduced to stably express luciferase (luc) and
GFP, as described below. Cell lines were harvested during the log phase of growth and seeded at a density of $0.2 \times 10^{6}$ cells $/ \mathrm{ml}$. Mononuclear cells in primary AML patient samples were harvested using Ficoll-Hypaque (Sigma-Aldrich) density gradient centrifugation.

RNA extraction and miRNA microarray analysis. Total RNA was extracted with miRNeasy Mini Kit (Qiagen) according to the manufacturer's protocol. RNA was labeled and hybridized on miRNA microarray chips as previously described (51). Microarray data have been deposited in GEO (accession no. GSE44828; http://www.ncbi.nlm.nih.gov/geo/query/acc. cgi?acc $=\mathrm{GSE} 44828)$.

RT-PCR analysis. RT reactions were carried out using $100 \mathrm{ng}$ total RNA with the TaqMan miRNA RT kit (Applied Biosystems) according to the manufacturer's instructions. Real-time PCR reactions were performed on a 7900 System (Applied Biosystems) using the TaqMan let-7a probe (Applied Biosystems) and gene-specific primers. For primary miRNA analysis, a 2-step qRT-PCR method was adopted using an NCode miRNA first-strand cDNA synthesis and qRT-PCR kit (Invitrogen). The real-time PCR reactions were then performed with a SYBR Green qPCR kit (Applied Biosystems) and the following gene-specific primers: pri-let-7a-1 forward, 5'-GATTCCTTTTCACCATTCACCCTGGATGTT-3'; pri-let-7a-1 reverse, 5'-TTTCTATCAGACCGCCTGGATGCAGACTTT-3'; pri-let-7a-2 forward, 5'-GCGGATCCTATGTTGTCTCTTATGAATGGCCC-3'; pri-let-7a-2 reverse, 5'-CGCTCGAGATCATGATCGTTCTCACCATGTTG-3'; pri-let-7a-3 forward, 5'-CGGAGTCCCATCGGCACCAAGACCGACTGC-3'; pri-let7a-3 reverse, 5'-TCTGTCCACCGCAGATATTACAGCCACTTC-3'.

In vitro transfection with synthetic miRNA. Synthetic let-7a and a scrambled oligonucleotide were purchased from Dharmacon (Thermo Scientific). In total, $2 \times 10^{6}$ OCI-AML3 cells were electroporated with $0.25 \mu \mathrm{M}$ oligonucleotide using Amaxa (Solution L, Program X001).

Lentiviral constructs and stably transduced cells. CXCR4 shRNA (pGIPZCXCR4-shRNA), YY1 shRNA (pGIPZ-YY1-shRNA), and NS shRNA control (pGIPZ-NS-shRNA) were purchased from OpenBiosystems for lentiviral constructs. The human codon-optimized firefly luciferase sequence was excised from pGL4.51 (Promega) and cloned into the pCDH-CMV-MCS-EF1-copGFP lentiviral vector to generate $\mathrm{pCDH}$ luc-CopGFP. The let-7a coding sequence was excised from the miRNA precursor construct pMIF-cGFP-Zeo-let-7a-2 (System Biosciences) and subcloned into the PGIPZ vector. Lentiviral infections were carried out according to standard procedures (52).

\section{Table 3}

Clinical and hematological parameters compared between AML patients with high and low CXCR4 MFI ratios

\begin{tabular}{lccr} 
Parameter & \multicolumn{2}{c}{ CXCR4 MFI ratio } & $P$ \\
& $>\mathbf{5}(\boldsymbol{n}=\mathbf{2 5})$ & $<\mathbf{5}(\boldsymbol{n}=\mathbf{2 5})$ & \\
Age $(\mathrm{yr})$ & $54.7 \pm 16.5$ & $58.8 \pm 16.8$ & 0.381 \\
PB blasts (\%) & $70.9 \pm 10.7$ & $64.9 \pm 16.9$ & 0.094 \\
wbc $(\times 109 / /)$ & $61.2 \pm 56.5$ & $38.5 \pm 34.7$ & $\mathbf{0 . 0 4 3}$ \\
LDH (U/l) & $918.5 \pm 875.2$ & $616.8 \pm 676.8$ & 0.150 \\
CD33 (\%) & $82.4 \pm 23.3$ & $66.9 \pm 32.3$ & 0.056 \\
CD34 (\%) & $33.0 \pm 36.8$ & $67.7 \pm 33.0$ & $\mathbf{0 . 0 0 2}$ \\
CD117 (\%) & $53.4 \pm 30.4$ & $80.2 \pm 18.9$ & $\mathbf{0 . 0 0 1}$ \\
CD49d (\%) & $93.7 \pm 7.7$ & $88.3 \pm 23.2$ & 0.337 \\
CXCR4 (\%) & $44.3 \pm 30.7$ & $7.0 \pm 4.8$ & $<\mathbf{0 . 0 0 1}$
\end{tabular}

Each value (mean $\pm \mathrm{SD}$ ) was determined at the time of diagnosis. $\mathrm{LDH}$, lactate dehydrogenase; $\mathrm{PB}$, peripheral blood. Significant $P$ values are denoted in bold. 
Lentiviral infections in AML primary samples were carried out following the standard protocol described by Kim and colleagues (53). In brief, 293T cells were cotransfected with pMD2.G, pCD-NL-BH, and pMIF-cGFPZeo-let-7a-2 lentiviral vectors using JetPrime transfection reagent (Polyplus) according to the manufacturer's protocol. Transfection medium was replaced after 12 hours with fresh DMEM with 10\% FBS, and 48 hours later, the viral supernatants were collected and concentrated using Centricon Plus-70 filter units (Millipore). AML patient samples were infected in the presence of $8 \mu \mathrm{g} / \mathrm{ml}$ Polybrene (Sigma-Aldrich), then cultured in STEM-EZ8 serum-free medium (Cellagen). At 4 days after infection, stably transduced leukemic cells were FACS sorted, resulting in a homogeneous population of $100 \% \mathrm{eGFP}^{+}$cells.

Flow cytometry. The expression of surface CXCR4 protein was analyzed by a LSR II flow cytometer (BD). Cells were incubated with saturating concentrations of allophycocyanin-conjugated anti-CXCR4 monoclonal antibody (12G5; BD Biosciences - Pharmingen) for 30 minutes at room temperature. The appropriate isotype-matched antibody was used as a negative control.

Western blot analysis. Cell lysates were separated on $10 \%$ polyacrylamide gels and transferred to Hybond-P membranes. The following antibodies were used: rabbit anti-human ITGB3, c-Myc, and BCL-XL (Cell Signaling Technology), and rabbit anti-human CXCR4 and YY1 (Abcam). GAPDH was used as the loading control.

Apoptosis analysis. Evaluation of apoptosis was performed using the previously described annexin $V$-propidium iodide binding assay (54). The extent of apoptosis was quantified as the percentage of annexin $\mathrm{V}^{+}$cells. To obtain absolute cell counts, 10,000 counting beads were added to each test tube before analysis by LSR II flow cytometer (BD). All experiments were conducted in triplicate.

In vivo Ara-C study in AML mouse models. NSG mice were intravenously injected with OCI3-luc-GFP $\left(1 \times 10^{6}\right.$ cells/100 $\left.\mu \mathrm{l}\right)$ or Molm13-luc-GFP $(2$ $\times 10^{6}$ cells $\left./ 100 \mu \mathrm{l}\right)$ leukemia cells. Bioluminescence imaging was used to monitor the tumor burden. Briefly, mice were anesthetized and imaged noninvasively with an in vivo imaging system (IVIS-200; Xenogen) after injection with luciferase substrate colenterazine (Biotium). Total body bioluminescence was quantified in a region of interest drawn around each mouse. Mice in treatment groups were given Ara-C $(100 \mathrm{mg} / \mathrm{kg}$ body weight) twice weekly, beginning 7 days after injection of leukemic cells. Selected mice with Molm13 cell engraftment from each group were humanely sacrificed by $\mathrm{CO}_{2}$ asphyxiation on day 18 , and the extent of leukemic infiltration was assessed by staining with an anti-firefly antibody (Promega). Spectral images were obtained using a Cambridge Research and Instrumentation attachment on an Olympus IX81 microscope with a disc-scanning unit confocal attachment, and images were analyzed using InForm software as described previously (55). 5 images per slide were quantified and averaged at 3 different focal depths within the tissue section.

For the primary human AML xenograft model, 32 female NSG mice were randomly divided into 4 groups, and $1 \times 10^{6} / 100 \mu$ let-7a-overexpressing cells as well as control cells were injected through tail vein. Retro-orbital bleeding was performed to monitor the leukemia burden in the mice. When the proportion of $\mathrm{hCD} 45^{+}$cells in peripheral blood mononuclear cells reached $1 \%$, mice in different groups were injected with either Ara-C (i.p., $100 \mathrm{mg} / \mathrm{kg}$ body weight, twice weekly) or the same volume of PBS.

Immunofluorescence staining and confocal microscopy. OCI-AML3 cells were fixed with $4 \%$ paraformaldehyde for 10 minutes, permeabilized with $0.5 \%$
Triton X-100 for 10 minutes, incubated with $5 \%$ bovine serum albumin for 1 hour, and then stained at room temperature for 2 hours with antiYY1 antibody (1:200; Abcam). Subsequently, cells were washed with PBS and incubated for 30 minutes with Alexa Fluor 594 goat anti-rabbit IgG (1:1,000; Invitrogen). After further washes with PBS and counterstaining with 4',6-diamidino-2-phenylindole solution, cells were spun onto slides and mounted with Dako fluorescent mounting medium. Cells were visualized and microphotographed under a FV1000 confocal laser-scanning microscope (Olympus).

ChIP assay. ChIP assays were performed with anti-YY1 antibody (ab12132; Abcam) and anti-H3K27 antibody (07-449; Millipore) as recommended by the manufacturer (EZ-Chip; Millipore). Genomic DNA pellets were resuspended in $20 \mu \mathrm{l}$ water. PCR was performed with $2 \mu \mathrm{l}$ immunoprecipitated material using primers encompassing the predicted YY1 binding sites in prilet-7a-1, pri-let-7a-2, and pri-let-7a-3 promoters. The sequences 5'-GATGATGAAGTAGTTGGACCTT-3' (forward) and 5'-CTGCCATTGAAATCTTGC-3' (reverse) were used to amplify via PCR the pri-let-7a-1 promoter fragment containing the predicted YY1 binding sites located at position -2,500. For the pri-let-7a-2 fragment containing the predicted YY1 consensus at positions $-1,920$ and $-1,800$, the sequences $5^{\prime}$-TGGTCACAGTAAGTGTTAAATCTTCTAC-3' (forward) and 5'-CTTACAGTTAAGGGATGATCATTTTT-3' (reverse) were used. Finally, the pri-let-7a-3 promoter fragment, encompassing a predicted YY1 binding site at position $-1,900$, was amplified using 5'-CTGTCCCTTCAGCCCTCAC-3' (forward) and 5'-CTGTCCCAGCATCATCTCG-3' (reverse). Products were analyzed using agarose gel and visualized with a GelDoc documentation system (Bio-Rad Laboratories).

Statistics. Unless otherwise indicated, results are mean \pm SEM from at least 3 independent experiments. 2-tailed Student's paired $t$ test was used for statistical comparisons between groups. For in vivo mouse experiments, overall survival curves and mean group survival times were estimated by the KaplanMeier method. $P$ values less than 0.05 were considered statistically significant.

Study approval. All animal work was done in accordance with a protocol approved by the Institutional Animal Care and Use Committee at The University of Texas MD Anderson Cancer Center. Samples from AML patients were collected after informed consent, as approved by the University of Texas MD Anderson Cancer Center Institutional Review Board and in accordance with the Declaration of Helsinki.

\section{Acknowledgments}

This work was supported by NIH grant AML P01 CA55164 and grants CA016672, CA143805, CA049639, CA136411, and CA100632 (to M. Andreeff); by a Paul and Mary Haas Chair in Genetics grant (to M. Andreeff); and by National Cancer Institute grant 1R01CA155056-01 (to M. Konopleva). POL6326 was obtained from Polyphor AG under MTA.

Received for publication August 28, 2012, and accepted in revised form March 7, 2013.

Address correspondence to: Michael Andreeff, Molecular Hematology and Therapy, Departments of Leukemia and Stem Cell Transplantation and Cellular Therapy, The University of Texas MD Anderson Cancer Center, 1515 Holcombe Blvd, Unit 0448, Houston, Texas 77030, USA. Phone: 713.792.7261; Fax: 713.563.7355; E-mail: mandreef@mdanderson.org.
1. Lowenberg B, Downing JR, Burnett A. Acute myeloid leukemia. NEngl J Med. 1999;341(14):1051-1062.

2. Estey E, Döhner H. Acute myeloid leukaemia. Lan cet. 2006;368(9550):1894-1907.

3. Konopleva MY, Jordan CT. Leukemia stem cells and microenvironment: biology and therapeutic targeting. J Clin Oncol. 2011;29(5):591-599.

4. Sipkins DA, et al. In vivo imaging of specialized bone marrow endothelial microdomains for tumour engraftment. Nature. 2005;435(7044):969-973.
5. Colmone A, Amorim M, Pontier AL, Wang S, Jablonski E, Sipkins DA. Leukemic cells create bone marrow niches that disrupt the behavior of normal hematopoietic progenitor cells. Science. 2008;322(5909):1861-1865. 
6. Konopleva M, Konoplev S, Hu W, Zaritskey AY, Afanasiev BV, Andreeff M. Stromal cells prevent apoptosis of AML cells by up-regulation of anti-apoptotic proteins. Leukemia. 2002;16(9):1713-1724.

7. Juarez J, Bradstock KF, Gottlieb DJ, Bendall LJ. Effects of inhibitors of the chemokine receptor CXCR4 on acute lymphoblastic leukemia cells in vitro. Leukemia. 2003;17(7):1294-1300.

8. Zeng $Z$, et al. Targeting the leukemia microenvironment by CXCR4 inhibition overcomes resistance to kinase inhibitors and chemotherapy in AML. Blood. 2009;113(24):6215-6224.

9. Chen Y, et al. Targeting CXCR4, SDF1, and $\beta$-adrenergic receptors in the AML microenvironment by novel antagonist POL6326, G-CSF, and isoproterenol. Blood. 2010;116:899-900.

10. He L, Hannon GJ. MicroRNAs: small RNAs with a big role in gene regulation. Nat Rev Genet. 2004;5(7):522-531.

11. Bartel DP. MicroRNAs: genomics, biogenesis, mechanism, and function. Cell. 2004;116(2):281-297.

12. Ambros V. MicroRNA pathways in flies and worms: growth, death, fat, stress, and timing. Cell. 2003;113(6):673-676

13. Lee RC, Feinbaum RL, Ambros V. The C. elegans heterochronic gene lin-4 encodes small RNAs with antisense complementarity to lin-14. Cell. 1993;75(5):843-854.

14. Lee YS, Dutta A. MicroRNAs in cancer. Annu Rev Pathol. 2009;4:199-227.

15. Meltzer PS. Cancer genomics: Small RNAs with big impacts. Nature. 2005;435(7043):745-746.

16. Visone R, Croce CM. MiRNAs and cancer. Am J Pathol. 2009;174(4):1131-1138.

17. Calin GA, et al. Frequent deletions and down-regulation of micro- RNA genes miR15 and miR16 at 13 q14 in chronic lymphocytic leukemia. Proc Natl Acad Sci U S A. 2002;99(24):15524-15529.

18. Garzon R, et al. MicroRNA gene expression during retinoic acid-induced differentiation of human acute promyelocytic leukemia. Oncogene. 2007;26(28):4148-4157.

19. O'Connell RM, et al. Sustained expression of microRNA-155 in hematopoietic stem cells causes a myeloproliferative disorder. J Exp Med. 2008;205(3):585-594.

20. Schotte D, et al. MicroRNA characterize genetic diversity and drug resistance in pediatric acute lymphoblastic leukemia. Haematologica. 2011;96(5):703-711.

21. Jongen-Lavrencic M, Sun SM, Dijkstra MK, Valk PJ, Lowenberg B. MicroRNA expression profiling in relation to the genetic heterogeneity of acute myeloid leukemia. Blood. 2008;111(10):5078-5085.

22. Marcucci G, et al. Prognostic significance of, and gene and microRNA expression signatures associated with, CEBPA mutations in cytogenetically normal acute myeloid leukemia with high-risk molecular features: a Cancer and Leukemia Group
B Study. J Clin Oncol. 2008;26(31):5078-5087.

23. Eisfeld A-K, et al. miR-3151 interplays with its host gene BAALC and independently impacts on outcome of patients with cytogenetically normal acute myeloid leukemia. Blood. 2012;120(2):249-258.

24. Farré D, et al. Identification of patterns in biological sequences at the ALGGEN server: PROMO and MALGEN. Nucleic Acids Res. 2003;31(13):3651-3653.

25. Atchison L, Ghias A, Wilkinson F, Bonini N, Atchison ML. Transcription factor YY1 functions as a PcG protein in vivo. EMBOJ. 2003;22(6):1347-1358.

26. de Nigris F, et al. CXCR4/YY1 inhibition impairs VEGF network and angiogenesis during malignancy. Proc Natl Acad Sci U S A. 2010; 107(32):14484-14489.

27. de Nigris F, et al. Deletion of Yin Yang 1 protein in osteosarcoma cells on cell invasion and CXCR4/ angiogenesis and metastasis. Cancer Res. 2008; 68(6): 1797-1808.

28. Gordon S, Akopyan G, Garban H, Bonavida B. Transcription factor YY1: structure, function, and therapeutic implications in cancer biology. Oncogene. 2005;25(8):1125-1142.

29. Tarnowski M, et al. Macrophage migration inhibitory factor is secreted by rhabdomyosarcoma cells, modulates tumor metastasis by binding to CXCR4 and CXCR7 receptors and inhibits recruitment of cancer-associated fibroblasts. Mol Cancer Res. 2010;8(10):1328-1343.

30. Wang H, et al. NF-אB-YY1-miR-29 regulatory circuitry in skeletal myogenesis and rhabdomyosarcoma. Cancer Cell. 2008;14(5):369-381.

31. Garzon R, Marcucci G, Croce CM. Targeting microRNAs in cancer: rationale, strategies and challenges. Nat Rev Drug Discov. 2010;9(10):775-789.

32. Marcucci G, Mrózek K, Radmacher MD, Garzon R, Bloomfield CD. The prognostic and functional role of microRNAs in acute myeloid leukemia. Blood. 2011;117(4):1121-1129.

33. Lima RT, Busacca S, Almeida GM, Gaudino G, Fennell DA, Vasconcelos MH. MicroRNA regulation of core apoptosis pathways in cancer. Eur J Cancer. 2011;47(2):163-174.

34. Nana-Sinkam SP, Croce CM. MicroRNAs as therapeutic targets in cancer. Transl Res. 2011; 157(4):216-225.

35. Rombouts EJC, Pavic B, Löwenberg B, Ploemacher RE. Relation between CXCR-4 expression, Flt 3 mutations, and unfavorable prognosis of adult acute myeloid leukemia. Blood. 2004;104(2):550-557.

36. Spoo AC, Lübbert M, Wierda WG, Burger JA. CXCR4 is a prognostic marker in acute myelogenous leukemia. Blood. 2007;109(2):786-791.

37. Uy GL, et al. A phase $1 / 2$ study of chemosensitization with the CXCR4 antagonist plerixafor in relapsed or refractory acute myeloid leukemia. Blood. 2012;119(17):3917-3924.

38. Pasquinelli AE, et al. Conservation of the sequence and temporal expression of let-7 heterochronic regulatory RNA. Nature. 2000;408(6808):86-89.

39. Reinhart BJ, et al. The 21-nucleotide let-7 RNA regulates developmental timing in Caenorhabditis elegans. Nature. 2000;403(6772):901-906

40. Shell S, et al. Let-7 expression defines two differentiation stages of cancer. Proc Natl Acad Sci U S A. 2007;104(27):11400-11405.

41. Shimizu S, et al. The let-7 family of microRNAs inhibits Bcl-xL expression and potentiates sorafenib-induced apoptosis in human hepatocellular carcinoma. J Hepatol. 2010;52(5):698-704.

42. Johnson SM, et al. RAS is regulated by the let-7 MicroRNA family. Cell. 2005;120(5):635-647.

43. Sampson VB, et al. MicroRNA let-7a down-regulates MYC and reverts MYC-induced growth in Burkitt lymphoma cells. Cancer Res. 2007;67(20):9762-9770.

44. Lee YS, Dutta A. The tumor suppressor microRNA let-7 represses the HMGA2 oncogene. Genes Dev. 2007;21(9):1025-1030.

45. Muller DW, Bosserhoff AK. Integrin [beta] 3 expression is regulated by let-7a miRNA in malignant melanoma. Oncogene. 2008;27(52):6698-6706.

46. Johnson CD, et al. The let-7 microRNA represses cell proliferation pathways in human cells. Cancer Res. 2007;67(16):7713-7722.

47. Arnold CP, et al. MicroRNA programs in normal and aberrant stem and progenitor cells. Genome Res. 2011;21(5):798-810.

48. Möhle R, et al. Functional response of leukaemic blasts to stromal cell-derived factor- 1 correlates with preferential expression of the chemokine receptor CXCR4 in acute myelomonocytic and lymphoblastic leukaemia. Br J Haematol. 2000;110(3):563-572.

49. Fierro FA, et al. Combining SDF-1/CXCR4 antagonism and chemotherapy in relapsed acute myeloid leukemia. Lenkemia. 2009;23(2):393-396.

50. Wozniak J, Kopec-Szlezak J. c-Kit receptor (CD117) expression on myeloblasts and white blood cell counts in acute myeloid leukemia. Cytometry B Clin Cytom. 2004;58(1):9-16.

51. Garzon R, et al. MicroRNA signatures associated with cytogenetics and prognosis in acute myeloid leukemia. Blood. 2008;111(6):3183-3189.

52. Chen Y, et al. Human extramedullary bone marrow in mice: a novel in vivo model of genetically controlled hematopoietic microenvironment. Blood. 2012;119(21):4971-4980.

53. Klemm L, et al. The B cell mutator AID promotes B lymphoid blast crisis and drug resistance in chronic myeloid leukemia. Cancer Cell. 2009;16(3):232-245.

54. Kojima K, et al. p53 activation of mesenchymal stromal cells partially abrogates microenvironment-mediated resistance to FLT3 inhibition in AML through HIF-1 $\alpha$-mediated down-regulation of CXCL12. Blood. 2011;118(16):4431-4439.

55 . Kidd S, et al. Origins of the tumor microenvironment: quantitative assessment of adipose-derived and bone marrow-derived stroma. PLoS One. 2012;7(2):e30563. 